\title{
Anytime System Level Verification via Parallel Random Exhaustive Hardware In the Loop Simulation is
}

\author{
Toni Mancini ${ }^{\mathrm{a}}$, Federico Mari ${ }^{\mathrm{a}}$, Annalisa Massini $^{\mathrm{a}}$, Igor Melatti ${ }^{\mathrm{a}}$, Enrico $_{\text {Tronci }}{ }^{\mathrm{a}}$ \\ ${ }^{a}$ Computer Science Department, Sapienza University of Rome, Italy
}

\begin{abstract}
System level verification of Cyber-Physical Systems has the goal of verifying that the whole (i.e., software + hardware) system meets the given specifications. Model checkers for hybrid systems cannot handle system level verification of actual systems. Thus, Hardware In the Loop Simulation (HILS) is currently the main workhorse for system level verification. By using model checking driven exhaustive HILS, System Level Formal Verification (SLFV) can be effectively carried out for actual systems.

We present a parallel random exhaustive HILS based model checker for hybrid systems that, by simulating all operational scenarios exactly once in a uniform random order, is able to provide, at any time during the verification process, an upper bound to the probability that the System Under Verification exhibits an error in a yet-to-be-simulated scenario (Omission Probability).

We show effectiveness of the proposed approach by presenting experimental results on SLFV of the Inverted Pendulum on a Cart and the Fuel Control System examples in the Simulink distribution. To the best of our knowledge, no previously published model checker can exhaustively verify hybrid systems of such a size and provide at any time an upper bound to the Omission Probability.
\end{abstract}

\footnotetext{
This paper is an extended and revised version of [1]

Email addresses: tmancini@di.uniroma1.it (Toni Mancini), mari@di.uniroma1.it (Federico Mari), massini@di.uniroma1.it (Annalisa Massini), melatti@di.uniroma1.it (Igor Melatti), tronci@di.uniroma1.it (Enrico Tronci)

Preprint submitted to Elsevier 


\section{Contents}

\begin{tabular}{lll}
\hline & Introduction & 3
\end{tabular}

1.1 Motivations . . . . . . . . . . . . . . . . . . . . 4

1.2 Main Contribution . . . . . . . . . . . . . . . . . . . . 5

1.3 Summary of Experimental Results . . . . . . . . . . . . . . . . . . . 6

1.4 Paper Outline . . . . . . . . . . . . . . . . . . 7

\begin{tabular}{|lll}
\hline 2 & Background and Preliminaries & $\mathbf{7}$
\end{tabular}

2.1 Modelling the Operational Environment . . . . . . . . . . . . . . . . . . 7

2.2 Modelling the Property to be Verified . . . . . . . . . . . . . . . . . . . . 9

2.3 Modelling the System Under Verification (SUVD) . . . . . . . . . . . . . . . 10

2.4 System Level Formal Verification $(\mathrm{SLFV})$. . . . . . . . . . . . . . . . . . 10

2.5 Parallel|HILS|Based Deterministic|SLFV] . . . . . . . . . . . . . . . . . . 11

$2.5 .1 \quad$ Disturbance Trace Generation and Splitting . . . . . . . . . . . . . 11

2.5.2 Computation of Optimised Simulation Campaigns . . . . . . . . . 12

3 Omission Probability 12

4 Enabling Omission Probability Computation: Random Exhaustive[SLFV 15

$\begin{array}{llr}5 & \text { Proof of Results } & 18\end{array}$

6 Experimental Results 20

6.1 Experimental Setting . . . . . . . . . . . . . . . . . . . . 20

6.1 .1 Case Studies . . . . . . . . . . . . . . . . . 20

6.1.1.1 Inverted Pendulum on a Cart (IPC) . . . . . . . . . . . . 20

$6.1 .1 .2 \quad$ Fuel Control System $(\mathrm{FCS})$. . . . . . . . . . . . . . . . 22

6.1 .2 Computational Infrastructure . . . . . . . . . . . . . . . 22

6.2 Disturbance Trace Generation and Splitting . . . . . . . . . . . . . . . . . 23

6.3 Computation of Optimised Simulation Campaigns . . . . . . . . . . . . . 23

6.4 Execution of the Simulation Campaigns . . . . . . . . . . . . . . . . 24

6.5 Overall Verification Time and Scalability . . . . . . . . . . . . . . . . . . . 27

6.5.1 Estimation of Sequential Verification Time . . . . . . . . . . . . . 27

6.5 .2 Speedup and Efficiency . . . . . . . . . . . . . . . . . . . . . . . . . . . 27

6.6 Omission Probability . . . . . . . . . . . . . . . . . . 28

6.7 Completion Time Estimation . . . . . . . . . . . . . . . . . . . . . . 28

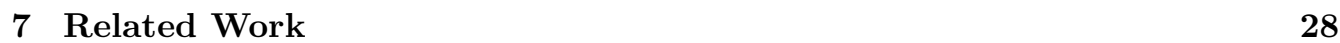

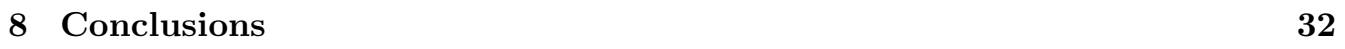

\begin{tabular}{|l|l|}
\hline A List of Acronyms & 35
\end{tabular} 


\section{Introduction}

The cost for fixing a design error in a system becomes larger and larger as the design proceeds from the requirement analysis to the implementation (see, e.g., 2, Chapter 1]) since the later in the design phase an error is detected the more reworking it may trigger. The above observation has motivated the development of methods and tools to verify correctness of a system already in the early phases of its design, namely during the requirement analysis or during the functional specification phases. The goal of all such approaches is to catch design errors well before the system implementation begins.

Of course, all such approaches are model based, that is they work on a model describing the system behaviour since no system implementation exists in the early design phases. Accordingly, System Verification is carried out by simulating a system model and analysing its behaviour under a suitable set of simulation scenarios.

For example, in a digital hardware setting, model based approaches have been used since a long time. In fact, even before considering going to silicon, a heavy simulation activity is performed, aimed at verifying that the system model (defined, e.g., using Verilog, VHDL or System ${ }^{1}$ ) meets the system requirements for most (possibly all) uncontrollable inputs (that is, primary inputs and faults the system is expected to withstand).

Along the same line of reasoning, in a purely software setting, before generating low level code, model based approaches are used to verify that the software model (defined, e.g., using AADL [3, 4]) meets the given requirements.

If all possible simulation scenarios are considered, then we can prove correctness of the system (i.e., absence of simulation scenarios violating the system requirements), otherwise we can only show that the system design is faulty (by exhibiting a simulation scenario violating the system requirements). In other words, a simulation campaign that does not consider all possible simulation scenarios can only show that the system design has a bug. To show correctness of the system design we need an exhaustive simulation campaign, that is one considering all possible simulation scenarios. A verification approach able to show system correctness is usually referred to as formal verification. One of the most successful techniques to carry out formal verification is Model Checking (see, e.g., [5]).

The need for model checking stems from the high cost that a bug may have for certain systems. This is the case for mission critical systems, that is, systems for which a system malfunctioning may entail loss of money, as well as for safety critical systems, that is, systems for which a system malfunctioning may entail loss of human lives. Examples of mission critical systems are: decision support systems, satellites, processors (e.g., the infamous P5 FDIV bug costed about $\$ 475$ million to INTEL). Examples of safety critical systems are: railway interlocking, avionics control software.

Many Cyber-Physical Systems ( $\overline{\mathrm{CPS}}$ ) are indeed mission or safety critical systems. Accordingly, in this paper we focus on formal verification techniques for CPS;

A CPS consists of hardware (e.g., motors, electrical circuits, etc) and software components. Thus, in order to verify a CPS design, we need methods and tools that can model and effectively support simulation of hardware as well as software components.

From a formal point of view, $\overline{C P S}$ s can be modelled as hybrid systems (see, e.g., [6] and citations thereof). Many Model Based Design software tools offer support for modelling

\footnotetext{
${ }^{1}$ http://www.mentor.com/products/fv/hdl_designer/
} 
and simulation of $\overline{C P S}$. Well known examples are Simulink ${ }^{2}$, VisSim ${ }^{3}$ and Modelice 4 All such tools take as input a (mathematical) model of the behaviour of the CPS along with a simulation scenario and provide as output the time evolution (trace or simulation run) of the system at hand.

System Level Verification of CPS aims at verifying that the whole (i.e., software + hardware) system meets the given specifications. System Level Formal Verification (SLFV) has the goal of exhaustively verifying that the above holds for all possible operational scenarios.

For digital circuits, formal verification is usually carried out using model checking techniques (e.g., see 7]). Unfortunately, model checkers for hybrid systems cannot handle SLFV of real world CPS;. Thus, Hardware In the Loop Simulation (HILS) is currently the main workhorse for system level verification of CPS $;$, and is supported by model based design tools (e.g., the previously mentioned Simulink, VisSim and Modelica).

In HILS, the actual software reads/sends values from/to mathematical models (simulation) of the physical systems (e.g., engines, analog circuits, etc.) it will be interacting with. Notwithstanding the word hardware, in HILS the only hardware present is the one devoted to support the system simulation, that is: computational and communication devices. This is because HILS is used in a model based design setting to validate the system design before any hardware is built (the whole goal of model based design). For example, Simulink, VisSim, Modelica, ESA Satellite Simulation Infrastructure SIMULUS 5 all provide simulation software supporting HILS, where the only hardware involved is just the computer on which the simulator is actually running.

Simulation can be very time consuming. Accordingly, in order to reduce system design time, Opal-RT ${ }^{6}$ and dSpace ${ }^{7}$ among others provide modelling and simulation software along with FPGA-based hardware to support real-time simulation. We note that in all cases the only hardware present in HILS is the one supporting the simulation itself.

\subsection{Motivations}

SLFV is an exhaustive HILS where all relevant simulation scenarios are considered. Using a parallel model checking driven approach, exhaustive HILS enables formal verification of actual systems. Examples of such systems are the Inverted Pendulum on a Cart (IPC) and the Fuel Control System (FCS) in the Simulink distribution (see Section 6.1.1.

Considering that parallel exhaustive HILS based SLFV may take days of computation (e.g., see [8]), from a practical point of view it would be very useful to have available at any time during the verification process, quantitative information about the degree of assurance attained. Such an information would enable us to evaluate if it is worth to continue the verification activity, or instead stop it since the degree of assurance attained can be considered adequate for the application at hand (graceful degradation).

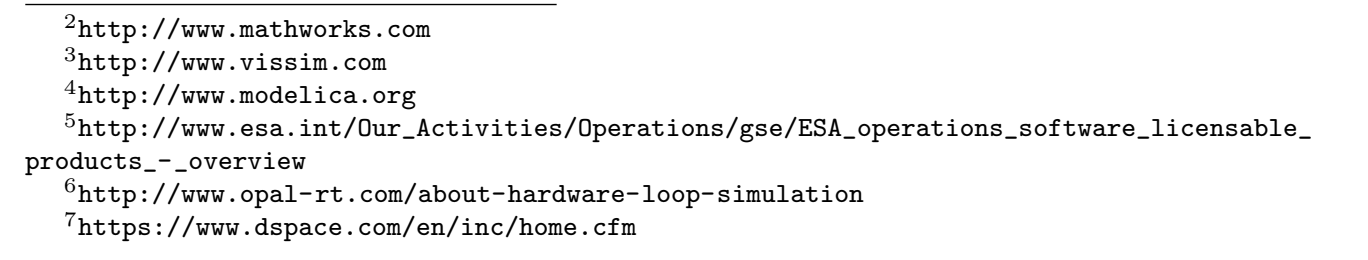


The above considerations suggest looking for a HILS based model checking approach satisfying the following requirements: (i) it is parallel, in order to make exhaustive HILS computationally feasible; (ii) it is exhaustive, since our focus is SLFV (iii) it is any time, to support graceful degradation.

The work in [9] presents a Propositional Satisfiability (SAT) based model checker for finite state systems which returns, at any time during the verification process, the coverage (i.e., the fraction of operational scenarios verified so far). Unfortunately, while coverage measures the amount of verification work done, it does not provide any information about the degree of assurance attained by the verification process. This is because formal verification aims at finding hard to find errors, i.e., errors that were not detected while verifying operational scenarios designed by experts. As a result, formal verification addresses the search of errors that we are unlikely to consider. For this reason, we can model the problem as an adversary system, that is a system where, knowing our verification strategy, the adversary places the error in operational scenarios we are less likely to visit. In such a framework, any deterministic ordering of the operational scenarios would not increase the degree of assurance until the end of the verification. In fact, the adversary would choose to place the single error in the last scenario picked by the verification procedure.

To provide a formally sound information about the degree of assurance attained by the verification process, approaches have been proposed which verify the operational scenarios in a random order. In particular, the work in 10. presents a Monte-Carlo based model checker for finite state systems that provides, at any time during the verification process, an upper bound to the probability that the System Under Verification (SUV) exhibits an error in a yet-to-be-simulated scenario (Omission Probability). The Omission Probability (OP) provides indeed the information we are looking for. Unfortunately, while Monte-Carlo based approaches guarantee randomness (thereby enabling OP computation) they are not exhaustive (within a finite time).

To the best of our knowledge, no model checker is available, neither for finite state systems nor for hybrid systems, which, at the same time, is both random and exhaustive, thereby enabling effective anytime SLFV. In this paper we advance the state of the art by presenting a parallel random exhaustive HILS based model checker along with experimental results showing its effectiveness.

\subsection{Main Contribution}

Our System Under Verification (SUV) is a Hybrid System (e.g., see [ $\underline{6}$ and citations thereof) whose inputs belong to a finite set of uncontrollable events (disturbances) modelling failures in sensors or actuators, variations in the system parameters, etc. We focus on deterministic systems (the typical case for control systems) and model nondeterministic behaviours (such as faults) with disturbances. Accordingly, in our framework, a simulation scenario is just a finite sequence of disturbances and a simulation campaign is a finite sequence of simulation instructions (namely: save a simulation state, restore a saved simulation state, remove a saved simulation state, inject a disturbance, advance the simulation of a given time length).

A system is expected to withstand all disturbance sequences that may arise in its operational environment. Correctness of a system is thus defined with respect to such admissible disturbance sequences. In our setting, the set of admissible disturbance sequences (disturbance model) can be defined as the language accepted by a suitable Finite 
State Automaton, which in turn can be defined using the modelling language of any finite state model checker.

In such a framework we address Bounded SLFV of safety properties. That is, given a time step $\tau$ (time quantum between disturbances) and a time horizon $T=\tau h$ we return PASS if there is no admissible disturbance sequence of length $h$ and time step $\tau$ that violates the given safety property. We return FAIL, along with a counterexample, otherwise. Therefore, SLFV is an exhaustive (with respect to admissible disturbance sequences) HILS, In other words, we are aiming at (black box) bounded model checking where the SUV behaviour is defined by a simulator (Simulink in our examples).

In such a setting, our main contribution can be summarised as follows. We present an anytime parallel random exhaustive HILS based model checker that effectively conjugates exhaustiveness with randomness, thereby enabling the computation of the Omission Probability.

While a simulation run for digital hardware takes order of milliseconds on a normal desktop computer, in our setting a simulation run takes order of seconds since it entails heavy numerical computations aimed at solving a system of many Ordinary Differential Equations (modelling the hardware components of the CPS). Indeed (see Section 6) simulation of operational scenarios takes almost $100 \%$ of the overall verification time. Resting on such observation we build on the SLFV approach discussed in 8 . In particular:

1. From the disturbance model we generate all admissible simulation scenarios and evenly split them into disjoint sets (slices).

2. For each slice, we compute a highly optimised simulation campaign that exploits simulator save/restore/remove commands in order to save on the simulation time while scheduling execution of all simulation scenarios exactly once and in a uniform random order. This guarantees exhaustiveness and allows us to compute, at any time during the verification process, an upper bound to the $\mathrm{OP}$

3. We run simulation campaigns in parallel. This guarantees a very efficient parallelism, since no communication among processes is needed. This step is supported by simulation tools (Simulink in our case study).

We also show that, thanks to the fact that we have first generated all admissible simulation scenarios, attaining point 2 above (i.e., anytime $\mathrm{OP}$ computation) can be done in a not-too-complicated (from both technical and computational points of view) and non-invasive way, by simply introducing a new module into the workflow of 8 , that seamlessly interoperates with the others.

\subsection{Summary of Experimental Results}

We implemented our approach and present (Section 6) experimental results on two case studies, namely the Inverted Pendulum on a Cart (IPC) and the Fuel Control System (FCS) examples in the Simulink distribution. Overall, we compute optimised simulation campaigns under four operational environments (disturbance models), which entail from 3208276 to 35641501 simulation scenarios.

Each processor (actually, a core of a 8-core machine) runs an instance of our (random) simulation campaign computation algorithm and takes as input a slice of our set of 
simulation scenarios. We present experimental results with 16,32, 64 machines totalling $128,256,512$ parallel processes. Our approach takes negligible time to generate an optimised simulation campaign for a given slice, with respect to the time needed to actually execute the simulation campaign (e.g., minutes vs. hours, see Section 6).

Our experimental results show that, by exploiting parallelism, our random exhaustive simulation campaigns effectively counteract the simulation time overhead due to randomisation. The above ensures feasibility of our parallel random exhaustive approach for actual systems, such as the Inverted Pendulum on a Cart (IPC) and Fuel Control System (FCS) examples in the Simulink distribution.

As for the Omission Probability ( $(\mathrm{OP})$, the worst case scenario is when just one error trace is present. Our experimental results (Section 6.6) show that, even in such a case, our upper bound to the $\mathrm{OP}$ decreases about linearly with respect to the coverage, that is the fraction of scenarios simulated. This is the best one can hope to obtain in our setting.

Finally, simulation of scenarios in random order allows us to use the coverage as a reliable estimator for the completion time of the whole verification task. Our experimental results show that the error in the completion time estimation decreases quickly with respect to coverage.

\subsection{Paper Outline}

Section 2 gives background notions to make our paper self-contained. Section 3 presents our formal framework, by formalising the notion of $\mathrm{OP}$ and by providing an upper bound for it, computable from the number of the yet-to-be-simulated traces in each slice. Section 4 outlines our algorithm which enables the computation, from a sequence (slice) of disturbance traces, a highly optimised simulation campaign which simulates the input traces in uniform random order and exploits the save/restore/remove capabilities of the simulator. Section 5 is devoted to the formal proofs of our results. Finally, Section 6 presents experimental results assessing effectiveness of our approach.

\section{Background and Preliminaries}

In this section we give some background notions. Unless otherwise stated, all definitions are based on [11, 8, 12]. Throughout the paper, we use $\mathbb{R}^{\geq 0}$ for the set of non-negative reals, $\mathbb{R}^{+}$for the set of strictly positive reals, and Bool $=\{0,1\}$ for the set of Boolean values ( 0 for false and 1 for true). $\mathbb{N}^{+}$denotes the set of positive natural numbers.

\subsection{Modelling the Operational Environment}

Our System Under Verification (SUV) is a Discrete Event System (DES), namely a continuous time Input-State-Output deterministic dynamical system [11 whose inputs are discrete event sequences. A discrete event sequence (Definition 1) is a function $u(t)$ associating to each (continuous) time instant $t \in \mathbb{R}^{\geq 0}$ a disturbance event (or, simply, disturbance). Disturbances, encoded by natural numbers in the interval $[0, d]$ (for a given $d \in \mathbb{N}^{+}$), represent uncontrollable events (e.g., faults). We use event 0 to represent the event carrying no disturbance. As no system can withstand an infinite number of disturbances within a finite time, we require that, in any time interval of finite length, 


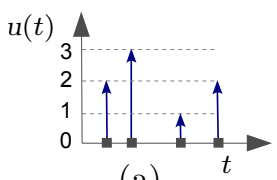

(a)

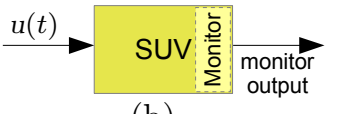

(b)

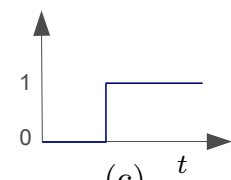

(c)

Figure 1: (a) A discrete event sequence $(d=3)$; (b) Our SUV embedding a monitor; (c) The SUV monitor output.

a discrete event sequence $u(t)$ differs from 0 only in a finite number of time points (Figure 1a).

Definition 1 (Discrete event sequence). Let $d \in \mathbb{N}^{+}$. A discrete event sequence over the natural interval $[0, d]$ is a function $u: \mathbb{R}^{\geq 0} \rightarrow[0, d]$ such that, for all $t \in \mathbb{R}^{\geq 0}$, the set $\left\{\tilde{t} \in \mathbb{R}^{\geq 0} \mid 0 \leq \tilde{t} \leq t\right.$ and $\left.u(\tilde{t}) \neq 0\right\}$ has finite cardinality. We denote with $\mathcal{U}_{d}$ the set of discrete event sequences over $[0, d]$ (following control engineering notation for input functions to dynamical systems, see e.g. [11]).

In Definition 2 and Definition 3 we specify the concepts of restriction and concatenation, respectively, for functions belonging to $\mathcal{U}_{d}$.

Definition 2. Let $\mathcal{U}_{d}$ be the set of discrete event sequences over the interval $[0, d]$. Given a function $u \in \mathcal{U}_{d}$ and two real numbers $0 \leq t_{1}<t_{2}$, we denote with $\left.u\right|_{\left[t_{1}, t_{2}\right)}$ the function $\left.u\right|_{\left[t_{1}, t_{2}\right)}:\left[t_{1}, t_{2}\right) \rightarrow[0, d]$, such that $\left.u\right|_{\left[t_{1}, t_{2}\right)}(t)=u(t)$ for all $t \in\left[t_{1}, t_{2}\right)$. We denote $\mathcal{U}_{d}^{\left[t_{1}, t_{2}\right)}$ the restriction of $\mathcal{U}_{d}$ to the domain $\left[t_{1}, t_{2}\right)$.

Definition 3. Assume that $t_{1}, t_{2}, t_{3} \in \mathbb{R}^{\geq 0}$ such that $t_{1}<t_{2}<t_{3}$. If $\omega \in \mathcal{U}_{d}^{\left[t_{1}, t_{2}\right)}$ and $\omega^{\prime} \in \mathcal{U}_{d}^{\left[t_{2}, t_{3}\right)}$, their concatenation, denoted as $\omega \omega^{\prime}$, is the function $\tilde{\omega} \in \mathcal{U}_{d}^{\left[t_{1}, t_{3}\right)}$ defined as:

$$
\tilde{\omega}(t)= \begin{cases}\omega(t) & \text { if } t \in\left[t_{1}, t_{2}\right) \\ \omega^{\prime}(t) & \text { if } t \in\left[t_{2}, t_{3}\right)\end{cases}
$$

System level verification follows an Assume-Guarantee approach aimed at showing that the SUV meets its specification (Guarantee) as long as the SUV operational environment behaves as expected (Assume). In this work we focus on bounded system level verification. As a consequence, we model (Definition 4) the SUV operational environment as the sequence of disturbances our SUV is expected to withstand within a finite time horizon, and we bound the time quantum between two consecutive disturbances.

Definition 4 (Disturbance trace). Let $h, d \in \mathbb{N}^{+}$. An $(h, d)$ disturbance trace $\delta$ is a finite sequence $\delta:[0, h-1] \rightarrow[0, d]$. Given $\tau \in \mathbb{R}^{+}$(time quantum), an $(h, d)$ disturbance trace $\delta$ is univocally associated to a discrete event sequence $u_{\delta}^{\tau}$, defined as follows: for all $t \in \mathbb{R}^{\geq 0}$, if there exists $j \in[0, h-1]$ such that $t=\tau j$, then $u_{\delta}^{\tau}(t)=\delta(j)$, else $u_{\delta}^{\tau}(t)=0$ (no disturbance).

Thus, a disturbance trace $\delta$ defines an operational scenario (namely, $u_{\delta}^{\tau}$ ) for our SUV Figure $2 \mathrm{~d}$ shows the discrete event sequence associated to a disturbance trace. We represent our [SUV] operational environment as a finite set of $(h, d)$ disturbance traces 


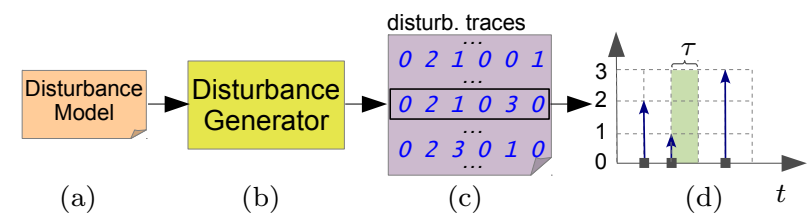

Figure 2: (a) Disturbance model; (b) CMurphi-based disturbance generator; (c) Generated sequence of disturbance traces $(d=3, h=6)$; (d) The discrete event sequence associated to the trace in the black rectangle in part (c), given time quantum $\tau$.

$\Delta=\left\{\delta_{0}, \ldots, \delta_{n-1}\right\}$, since $U_{\Delta}^{\tau}=\left\{u_{\delta_{0}}^{\tau}, \ldots, u_{\delta_{n-1}}^{\tau}\right\}$ (for a given $\tau \in \mathbb{R}^{+}$) defines the operational scenarios our SUV] should withstand. Note that, by taking $h$ large enough (as in Bounded Model Checking (BMC) ) and $\tau$ small enough (to faithfully model our SUV operational scenarios), we can achieve any desired precision. On such considerations rests the effectiveness of the approach.

As it is typically infeasible to define a SUV operational environment by explicitly listing all its disturbance traces, we define an operational environment with a disturbance model which is in turn defined as the language accepted by a suitable Finite State Automaton. The following example illustrates this point.

Example 1. Consider a disturbance model consisting of one disturbance (namely, a fault) which is always recovered within 4 seconds. Let us assume that between two consecutive disturbances (faults) there must be at least 5 seconds and that disturbances can arise only at time steps multiple of $\tau=1$ second (time quantum). We also assume that the verification time horizon is set to 6 seconds.

In Figure 3a we show disturbance traces represented as strings of zeros (no disturbance) and ones (disturbance), with time flowing from left to right. The 8 strings terminated by $\sqrt{ }$ denote all the disturbance traces accepted by the disturbance model (admissible disturbance traces). The 14 strings terminated by $\otimes$ are the shortest non-admissible sequences of disturbances, that is disturbance sequences that cannot be extended to admissible disturbance traces.

Figure 36 shows the pseudo-code for a finite state automaton recognising such a language.

We define a finite state automaton for a disturbance model using the modelling language of a finite state model checker (namely, CMurphi [13]), along the lines of [8].

\subsection{Modelling the Property to be Verified}

Along the lines of [14, we model the property to be verified with a continuous-time monitor which observes the state of the system to be verified and checks whether the property under verification is satisfied (Figure $1 \mathrm{~b}$ ). The output of the monitor is 0 as long as the property under verification is satisfied and becomes and stays 1 (sustain) as soon as the property fails, thus ensuring that we never miss a property failure report, even when sampling the monitor output only at discrete time points (Figure 1 ). The use of monitors gives us a flexible approach to model the property to be verified. In particular, it is easy to model bounded safety and bounded liveness properties as monitors. Figures 8 and 9 show the Simulink/Stateflow representations of our two case studies (Inverted 


$\begin{array}{ll}000000 \sqrt{ } & 010000 \sqrt{ } \\ 000001 \sqrt{ } & 010001 \otimes \\ 000010 \sqrt{ } & 01001 \otimes \\ 000011 \otimes & 0101 \otimes \\ 000100 \sqrt{ } & 011 \otimes \\ 000101 \otimes & 100000 \sqrt{ } \\ 00011 \otimes & 100001 \sqrt{ } \\ 001000 \sqrt{ } & 10001 \otimes \\ 001001 \otimes & 1001 \otimes \\ 00101 \otimes & 101 \otimes \\ 0011 \otimes & 11 \otimes\end{array}$

(a) Admissible disturbance traces $(\sqrt{ })$ and shortest disturbance sequences that cannot be extended to an admissible disturbance trace $(\otimes)$

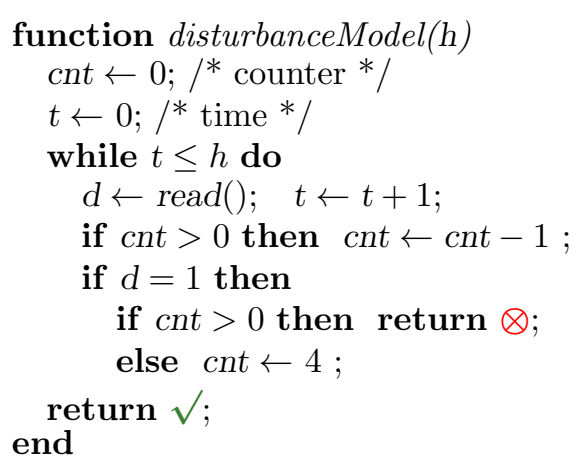

(b) Finite state automaton recognising the language of admissible disturbance traces (disturbance model)

Figure 3: Example 1

Pendulum on a Cart and Fuel Control System, respectively), along with their property monitors (see Section 6).

\subsection{Modelling the $\widehat{S U V}$}

Since the monitor output is all we need to carry out our verification task, we can model our SUV along with the property to be verified as a DES with an embedded monitor (Figure 1 $1 \mathrm{~b}$ ). We call Monitored Discrete Event System (MDES) such a DES,

According to our black-box approach to SUV] modelling, given a time quantum $\tau \in$ $\mathbb{R}^{+}$, Definition 5 formalises an $(h, d)$ MDES as a function $\mathcal{H}$ associating, to each $(h, d)$ disturbance trace $\delta$, a Boolean value $\mathcal{H}(\delta)$ representing the output of the SUV monitor at time $T=\tau h$ (the time horizon), when the system (starting from its initial state) is given as input the discrete event sequence $u_{\delta}^{\tau}(t)$ associated to $\delta$. For any disturbance trace $\delta$, $\mathcal{H}(\delta)$ is 1 (error) if and only if $u_{\delta}^{\tau}(t)$ violates the property under verification within time horizon $T=\tau h$ (with the SUV starting from its initial state).

Definition $5\left((h, d)\right.$ Monitored (DES). Let $h, d \in \mathbb{N}^{+} . A(h, d)$ Monitored Discrete Event System (MDES) is a function $\mathcal{H}:([0, h-1] \rightarrow[0, d]) \rightarrow$ Bool mapping all $(h, d)$ disturbance traces to Boolean values.

\subsection{System Level Formal Verification SLFV}

Definition 6 formalises our bounded System Level Formal Verification problem.

Definition 6. A System Level Formal Verification (SLFV) problem is a tuple $P=(h$, $d, \Delta, \mathcal{H})$ where: $h, d \in \mathbb{N}^{+}, \Delta=\left\{\delta_{0}, \ldots, \delta_{n-1}\right\}$ is an $(h, d)$ set of disturbance traces, and $\mathcal{H}$ is a $(h, d)$ MDES.

The answer to SLFV problem $P$ is FAIL if there exists a disturbance trace $\delta$ in $\Delta$ such that $\mathcal{H}(\delta)=1$ (in such a case also the counterexample $\delta$ is returned), PASS otherwise. 


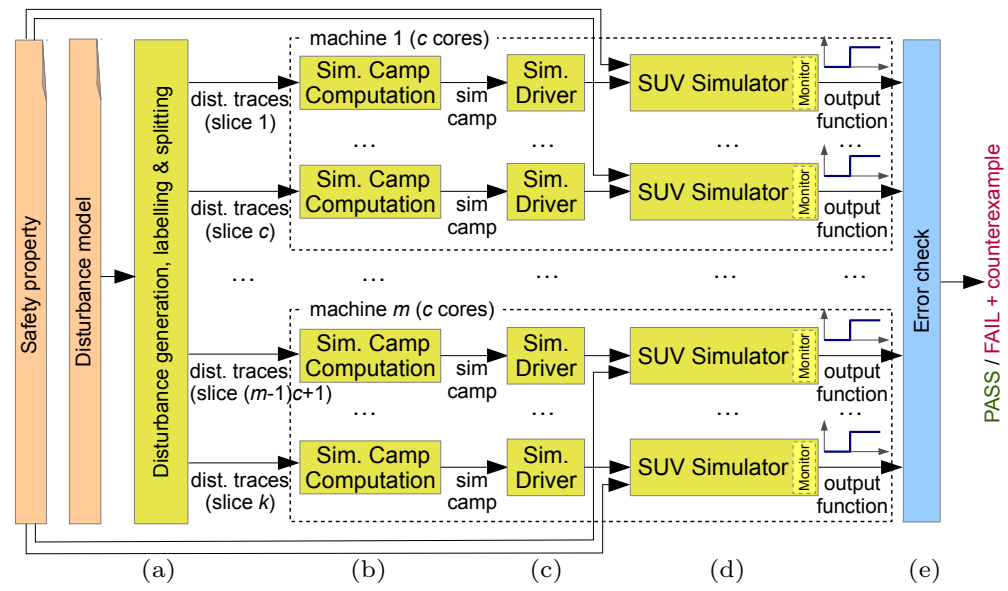

Figure 4: Parallel HILS based dSLFV 8]: $k$ parallel processes are run on $m$ multi-core machines (we show a possible deployment with machines having $c$ cores each, i.e., $k=m c$ ).

Note that, notwithstanding the fact that the number of states of our [SUV is infinite and we are in a continuous time setting, to answer a SLFV problem we only need to check a finite number of disturbance traces. This is because we are bounding: (a) our time horizon to $T=\tau h$, and (b) the set of time points at which disturbances can take place, by taking $\tau$ as the time quantum among disturbance events.

\subsection{Parallel HILS Based Deterministic SLFV}

In the black-box parallel approach shown in 8], the MDES $\mathcal{H}$ defining our SUV (plus the property to be verified) is defined using the modelling language of a suitable simulator (e.g., MatLab and Stateflow for Simulink). The answer to a SLFV problem $(h, d, \Delta, \mathcal{H})$ is computed by simulating each operational scenario $\delta$ in the operational environment $\Delta$, thus by performing an exhaustive (with respect to $\Delta$ ) Hardware In the Loop Simulation (HILS). The overall workflow is shown in Figure 4 and described in the remainder of this section. We will refer to this approach as Deterministic SLFV (dSLFV), where the word "deterministic" stems from the fact that the disturbance traces are verified in a deterministic order.

\subsubsection{Disturbance Trace Generation and Splitting}

Our CMurphi-based trace generator (see Section 2.1 and Figure 4a) works in DepthFirst Search (DFS) mode, and, given the set of disturbances, produces a sequence $\Delta$ of $n$ disturbance traces. Each generated trace $\delta$ in $\Delta$ is annotated with labels and is of the form $\delta=\left(l_{0}, d_{0}, l_{1}, d_{1}, \ldots, l_{h-1}, d_{h-1}, l_{h}\right)$, where $\delta=\left(d_{0}, \ldots, d_{h-1}\right)$ is a sequence of disturbances satisfying the disturbance model and $l_{0}, \ldots, l_{h}$ belong to a countable set of labels $L$.

Labels are defined by an injective map $\lambda$ from finite sequences of disturbances (including the empty sequence) to $L$. Label $l_{0}$ is common to all traces and it is associated to the simulator initial state. Prefixes of disturbance sequences $\left(\hat{d}_{0}, \ldots, \hat{d}_{p-1}\right)$ common 
to multiple disturbance traces in $\Delta$ are followed by the same label $\hat{l}_{p}=\lambda\left(\hat{d}_{0}, \ldots, \hat{d}_{p-1}\right)$. Figure 5a shows a short sequence of labelled disturbance traces.

Labels identifying common disturbance prefixes are essential in the efficient computation of optimised simulation campaigns. Note that, given that our CMurphi-based generator runs in DFS mode, disturbance traces can be labelled at no additional computational cost during generation. Trace labelling during generation greatly increases the efficiency of the simulation campaign optimiser, as shown in 8 .

In the following, we will use $\delta^{\lambda}$ instead of $\delta$ (respectively, $\Delta^{\lambda}$ instead of $\Delta$ ) when we want to emphasise that a trace $\delta$ is annotated (respectively, all traces in set $\Delta$ are annotated) with labels, or when we need such labels. In order to enable parallel verification via $k \in \mathbb{N}^{+}$processes, we evenly partition the sequence of labelled disturbance traces $\Delta^{\lambda}$ into $k \in \mathbb{N}^{+}$sequences of disturbance traces $\Delta^{\lambda}{ }_{0}, \ldots, \Delta^{\lambda}{ }_{k-1}$ (called slices).

The splitting process produces slices containing $\lceil n / k\rceil$ traces each, except the last slice, which may contain fewer traces if $n$ is not a multiple of $k$.

\subsubsection{Computation of Optimised Simulation Campaigns}

In the next phase of the workflow in Figure 4 , we use the $k$ slices $\Delta^{\lambda}{ }_{0}, \ldots, \Delta^{\lambda} k-1$ generated so far to compute, independently and in parallel, $k$ highly optimised simulation campaigns (Figure 4b). Such simulation campaigns can be simulated, again independently and in parallel, using $k$ simulators, each one running (e.g., on a different core of a bunch of multi-core machines) a model for $\mathcal{H}$ (Figure $4 c-\mathrm{d}$ ).

The answer to the SLFV problem is FAIL if one of the simulation campaigns raises the simulator output function to 1 (in this case the disturbance trace $\delta$ which raised the error is returned as a counterexample, see Figure 4 4 ). The answer is PASS otherwise.

Each simulator accepts four basic commands: store, load, free, run. Command store $(l)$ stores in memory the current state of the simulator and labels with $l$ such a state. Command $\operatorname{load}(l)$ loads into the simulator the stored state labelled with $l$. Command free $(l)$ removes from the memory the state labelled with $l$. Command run $(e, t)$ (with $e \in[0, d]$ and $t \in \mathbb{R}^{+}$) injects disturbance $e$ and then advances the simulation of time $t$. A simulation campaign is thus a sequence of simulator commands.

The simulation campaign $\chi_{i}(0 \leq i<k)$ computed from input slice $\Delta^{\lambda}{ }_{i}$ steers the simulator as to execute all disturbance traces in $\Delta^{\lambda}{ }_{i}$ according to their order in the slice (this is the reason why we refer to this approach as Deterministic SLFV).

Each disturbance trace is executed as if starting from the simulator initial state. However, by using commands store and load, the optimiser avoids revisiting simulation states as much as possible (as in explicit model checking). Using command free the optimiser removes from the simulator memory states that will never be needed in the remaining part of the simulation campaign. This is needed since each state may require many KB of memory (150-300 KB in the case studies presented in this paper).

Figure $5 \mathrm{~b}$ shows the optimised simulation campaign computed from the sequence of labelled disturbance traces in Figure 5a, in the simple case where we ignore any limit on number of states that can be kept simultaneously stored in the simulator memory.

\section{Omission Probability}

This section formally defines the notion of Omission Probability (OP) (Definitions 7 and 8) and provides an upper bound for it, which can be computed anytime during the 


$$
\begin{aligned}
& \text { a0b2c1d0e0f1g } \\
& \text { a0b2c2h0i0j0k } \\
& \text { a0b2c2h0i3m0n } \\
& \text { a0b2c3p1q1r0s } \\
& \text { a0b2c3p2v2w0x } \\
& \text { a0b3y0z0 } \alpha 1 \beta 0 \lambda
\end{aligned}
$$

(a) A sequence of labelled disturbance traces (labels in red, disturbances in blue).

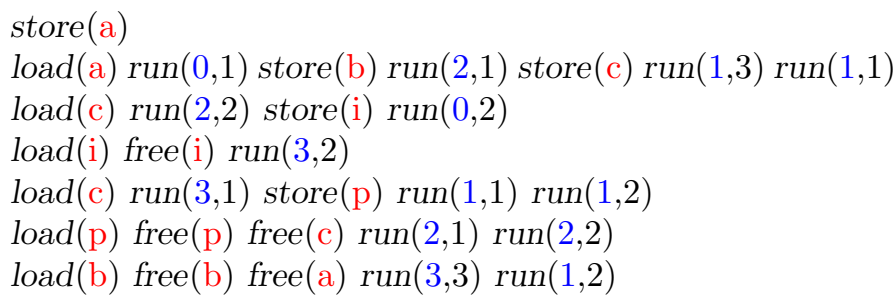

(b) Optimised simulation campaign which simulates the sequence of disturbance traces in (a) in their natural order.

Figure 5: Labelled disturbance traces and optimised simulation campaign.

parallel verification process from the number of the yet-to-be-simulated traces in each slice (Theorem 1 ).

Notation 1 (Set of permutations of a set). Let $\Delta=\left\{\delta_{0}, \ldots, \delta_{n-1}\right\}$ be a finite non-empty set. We denote with $\operatorname{Perm}(\Delta)$ the set of permutations of elements of $\Delta$ :

$\operatorname{Perm}(\Delta)=\left\{\left(\theta_{0}, \ldots, \theta_{n-1}\right) \mid\left(\forall i \in[0, n-1] \theta_{i} \in \Delta\right) \wedge\left(\forall i, j \in[0, n-1] i \neq j \rightarrow \theta_{i} \neq \theta_{j}\right)\right\}$

If $\hat{\Delta}=\left(\delta_{0}, \ldots, \delta_{n-1}\right) \in \operatorname{Perm}(\Delta)$ we write also $\hat{\Delta}(i)$ for $\delta_{i}$.

A Random Sequence Generator (RSG) models the extraction of a random permutation from a given finite non-empty set (which, in our case, will be the set of admissible disturbance traces $\Delta$ ). This is formalised in Definition 7 .

Definition 7 (Random Sequence Generator). Let $\Delta$ be a finite non-empty set. A Random Sequence Generator (RSG) for $\Delta$ is a probability space $(\Omega, \mathcal{F}, \operatorname{Pr})$, where:

- $\Omega$ (the space of outcomes) is the set of permutations of $\Delta$, that is $\Omega=\operatorname{Perm}(\Delta)$.

- $\mathcal{F}$ (the space of events) is the set of subsets of $\Omega$, that is: $\mathcal{F}=2^{\Omega}=\{E \mid E \subseteq \Omega\}$.

- $\operatorname{Pr}: \mathcal{F} \rightarrow[0,1]$ is a probability measure such that, for all $\omega \in \Omega, \operatorname{Pr}(\omega)=\frac{1}{|\Delta| !}$. That is, permutations of $\Delta$ are extracted with uniform probability. Since $\Omega$ is countable (actually finite), the probability of any event $E \in \mathcal{F}$ is defined as $\operatorname{Pr}(E)$ $=\sum_{\omega \in E} \operatorname{Pr}(\omega)$.

Let $\left(\Delta_{0}, \ldots, \Delta_{k-1}\right)$ be a partition of $\Delta$ into $k \in \mathbb{N}^{+}$disjoint non-empty sets. For any $0 \leq i<k$, let $\left(\Omega_{i}, \mathcal{F}_{i}, \operatorname{Pr}_{i}\right)$ be an $R S G$ for $\Delta_{i}$. A Random Sequence Generator for $\left(\Delta_{0}, \ldots, \Delta_{k-1}\right)$ is a probability space $(\Omega, \mathcal{F}, \operatorname{Pr})$, where: $\Omega=\times_{i=0}^{k-1} \Omega_{i}, \mathcal{F}=\times_{i=0}^{k-1} \mathcal{F}_{i}$ and, for each event $E_{0} \times \cdots \times E_{k-1} \in \mathcal{F}\left(E_{i} \in \mathcal{F}_{i}\right.$ for each $\left.0 \leq i<k\right), P\left(E_{0} \times \cdots \times E_{k-1}\right)=$ $\prod_{i=0}^{k-1} \operatorname{Pr}_{i}\left(E_{i}\right)$

Note that, by Definition 7 a RSG for a partition $\left(\Delta_{0}, \ldots, \Delta_{k-1}\right)$ of $\Delta$ models the extraction of $k$ permutations of, respectively, $\Delta_{0}, \ldots, \Delta_{k-1}$. For all $0 \leq i<k$, the extracted permutation of $\Delta_{i}$ is chosen uniformly among all possible permutations of $\Delta_{i}$. Also, the $k$ permutations are extracted independently from each other. 
Definition 8 defines the probability of omitting the simulation of a trace $\bar{\delta} \in \Delta$ containing an error (i.e., $\mathcal{H}(\bar{\delta})=1$ ) when the verification process has already examined $q_{i}$ disturbance traces, where $q_{i} \in\left\{0, \ldots,\left|\Delta_{i}\right|\right\}$, from a random permutation of slice $\Delta_{i}$, for all $0 \leq i<k$. Thus $q_{i}$ represents the state of advancement of the computation on the $i$-th slice $\Delta_{i}$. Hence $\left(q_{0}, \ldots, q_{k-1}\right)$ defines the state of advancement (stage) of the computation on all slices. We denote this probability as Omission Probability (OP).

Definition 8 (Omission Probability). Let $(h, d, \Delta, \mathcal{H})$ be a System Level Formal Verification SLFV) problem and $\left(\Delta_{0}, \ldots, \Delta_{k-1}\right)$ be a partition of $\Delta$ into $k \in \mathbb{N}^{+}$disjoint non-empty sets. Let $(\Omega, \mathcal{F}, \operatorname{Pr})$ be an RSG for $\left(\Delta_{0}, \ldots, \Delta_{k-1}\right)$, and $\left(q_{0}, \ldots, q_{k-1}\right)$ a tuple such that $q_{i} \in\left\{0, \ldots,\left|\Delta_{i}\right|\right\}$ for each $0 \leq i<k$.

The Omission Probability (OP) for $\left(\Delta_{0}, \ldots, \Delta_{k-1}\right)$ at stage $\left(q_{0}, \ldots, q_{k-1}\right)$, is defined as:

$\widetilde{O P}_{\mathcal{H}}\left(\left|\Delta_{0}\right|, \ldots,\left|\Delta_{k-1}\right|, q_{0}, \ldots, q_{k-1}\right)=\operatorname{Pr}\left(\left\{\begin{array}{l|l}\left(\omega_{0}, \ldots, \omega_{k-1}\right) & \begin{array}{l}\forall i \in[0, k-1] \omega_{i} \in \Omega_{i} \wedge \\ A B\left(\left(\omega_{0}, \ldots, \omega_{k-1}\right),\left(q_{0}, \ldots, q_{k-1}\right)\right)\end{array}\end{array}\right\}\right)$ where:

- $A B$ is defined as $A\left(\left(\omega_{0}, \ldots, \omega_{k-1}\right),\left(q_{0}, \ldots, q_{k-1}\right)\right) \wedge B\left(\left(\omega_{0}, \ldots, \omega_{k-1}\right),\left(q_{0}, \ldots, q_{k-1}\right)\right)$

- $A$ (After) is: $A\left(\left(\omega_{0}, \ldots, \omega_{k-1}\right),\left(q_{0}, \ldots, q_{k-1}\right)\right)=\exists i \in[0, k-1] \exists j \in\left[q_{i},\left|\Delta_{i}\right|\right] \mathcal{H}\left(\omega_{i}(j)\right)=$ 1 ;

- $B$ (Before) is: $B\left(\left(\omega_{0}, \ldots, \omega_{k-1}\right),\left(q_{0}, \ldots, q_{k-1}\right)\right)=\forall i \in[0, k-1] \forall j \in\left[0, q_{i}-\right.$ 1] $\mathcal{H}\left(\omega_{i}(j)\right)=0$.

In Definition 8 , formula $A$ (After) states that there exists a yet-to-be-simulated trace $\bar{\delta}$ (some trace $j \geq q_{i}$ of some slice $i$ ) containing an error, i.e., such that $\mathcal{H}(\bar{\delta})$ evaluates to 1 . Formula $B$ (Before) states that none of the already simulated traces contains an error, i.e., function $\mathcal{H}$ evaluates to 0 for all of them.

The following Theorem 1 gives an upper bound to the $\mathrm{OP}$, after having simulated $q_{i}$ randomly extracted traces from slice $\Delta_{i}$ (for each $0 \leq i<k$ ). In particular, Theorem 1 provides a bound to the probability of omitting the simulation of a trace $\bar{\delta} \in \Delta$ containing an error when the verification process has already examined $q_{i}$ disturbance traces from the (the permutation of) slice $\Delta_{i}$ (for all $0 \leq i<k$ ). Importantly, the bound provided does not depend on $\mathcal{H}$, i.e., it is independent of the system model. The proof of the theorem is in Section 5

Theorem 1. Let $(h, d, \Delta, \mathcal{H})$ be a $S L F V$ problem and $\left(\Delta_{0}, \ldots, \Delta_{k-1}\right)$ be a partition of $\Delta$ into $k \in \mathbb{N}^{+}$disjoint non-empty sets. Let $(\Omega, \mathcal{F}, \operatorname{Pr})$ be a Random Sequence Generator for $\left(\Delta_{0}, \ldots, \Delta_{k-1}\right)$ and $\left(q_{0}, \ldots, q_{k-1}\right)$ a tuple such that $q_{i} \in\left\{0, \ldots,\left|\Delta_{i}\right|\right\}$ for each $0 \leq i<k$. We have:

$$
{ }_{O P}\left(\left|\Delta_{0}\right|, \ldots,\left|\Delta_{k-1}\right|, q_{0}, \ldots, q_{k-1}\right) \leq 1-\min \left\{\frac{q_{i}}{\left|\Delta_{i}\right|} \mid 0 \leq i<k\right\}
$$

Note that the construction of the slices $\Delta_{0}, \ldots, \Delta_{k-1}$ from $\Delta$ is non-deterministic (i.e., any partitioning of $\Delta$ would work), whereas, for each slice, the selection of a permutation 
is a probabilistic process, modelled as a $\mathrm{RSG}$, Accordingly, Theorem 1 bounds the $\mathrm{OP}$ using the worst case distribution, i.e., the distribution yielding the greatest $\mathrm{OP}$ From this stems the min function in the right member of the inequality in Theorem 1

Finally, we observe that, from Theorem 1 , it follows that $\widehat{\mathrm{OP}}_{\mathcal{H}}\left(\left|\Delta_{0}\right|, \ldots,\left|\Delta_{k-1}\right|,\left|\Delta_{0}\right|, \ldots,\left|\Delta_{k-1}\right|\right)=$ 0 , that is, our verification task terminates after $\max \left\{\left|\Delta_{i}\right| \mid 0 \leq i<k\right\}$ parallel steps, having simulated all traces in $\Delta$.

\section{Enabling Omission Probability Computation: Random Exhaustive SLFV}

Our disturbance trace generator (see Section 2.1) produces a sequence of disturbance traces $\Delta^{\lambda}$, whose order is deterministically chosen by the employed algorithm (in our case, Depth-First Search (DFS $)$ ). As a consequence, no information about the Omission Probability (OP) can be inferred during the verification process if the simulation campaign computed from each slice verifies the sequence of disturbance traces therein according to their order (as done by the Deterministic SLFV (dSLFV) approach of Section 2.5), or according to any deterministic order, as argued in Section 1.1 .

From Section 3 it follows that it is possible to enable $\mathrm{OP}$ computation (and hence, graceful degradation during the computationally very expensive simulation phase) by simulating the disturbance traces within each slice in an order uniformly chosen at random.

Here we show how we can add support to $\mathrm{OP}$ computation in the workflow of Figure 4 in a not-too-complicated and non-invasive way, by introducing an additional step, in the parallel portion of the workflow, which implements a Random Sequence Generator (RSG).

The new workflow, which we refer to as Random Exhaustive SLFV (rSLFV), is shown in Figure 6. All slices are given as input, in parallel, to instances the new Random Sequence Generator module, shown as Algorithm 1 . The Random Sequence Generator module reads a sequence of disturbance traces and computes a random permutation of it, uniformly chosen among all possible permutations, thus implementing a RSG (Definition 7). We argue that the introduction of the new Random Sequence Generator module is non-too-complicated for what concerns both its implementation (Algorithm 1) and its computational viability (see Section 6.3). It is also non-invasive, as it seamlessly interoperates with the dSLFV workflow of Section 2.5

As the input sequence can be too large to be kept in main memory, the Random Sequence Generator module implements a disk-based multi-round algorithm (shown as Algorithm 11 which takes efficiency into account by using, in each round, as much main memory as possible and by reading/writing the input/output trace files sequentially.

Let $n=\left|\Delta^{\lambda}\right|$ be the number of disturbance traces in the input sequence. Given parameter $z \in \mathbb{N}^{+}$for the maximum number of disturbance traces which can be simultaneously stored in main memory, the algorithm, at each round $r \geq 1$, selects the $z$ traces which will have output positions in the interval $[(r-1) z, \min (n, r z-1)]$.

The above selection is performed by computing the first $z$ elements of a random permutation of the traces not yet in the output file $\Delta_{\text {rnd }}^{\lambda}$, chosen uniformly among all possible permutations. Such a permutation prefix is computed by function rndPermPrefix () (Algorithm 1, from line 20). Function rndPermPrefix() performs a swap-based computation of a permutation of integers $\left[0, \mid \Delta^{\lambda}\right.$ in $\left.\mid-1\right]$ (uniformly chosen at random) and interrupts 


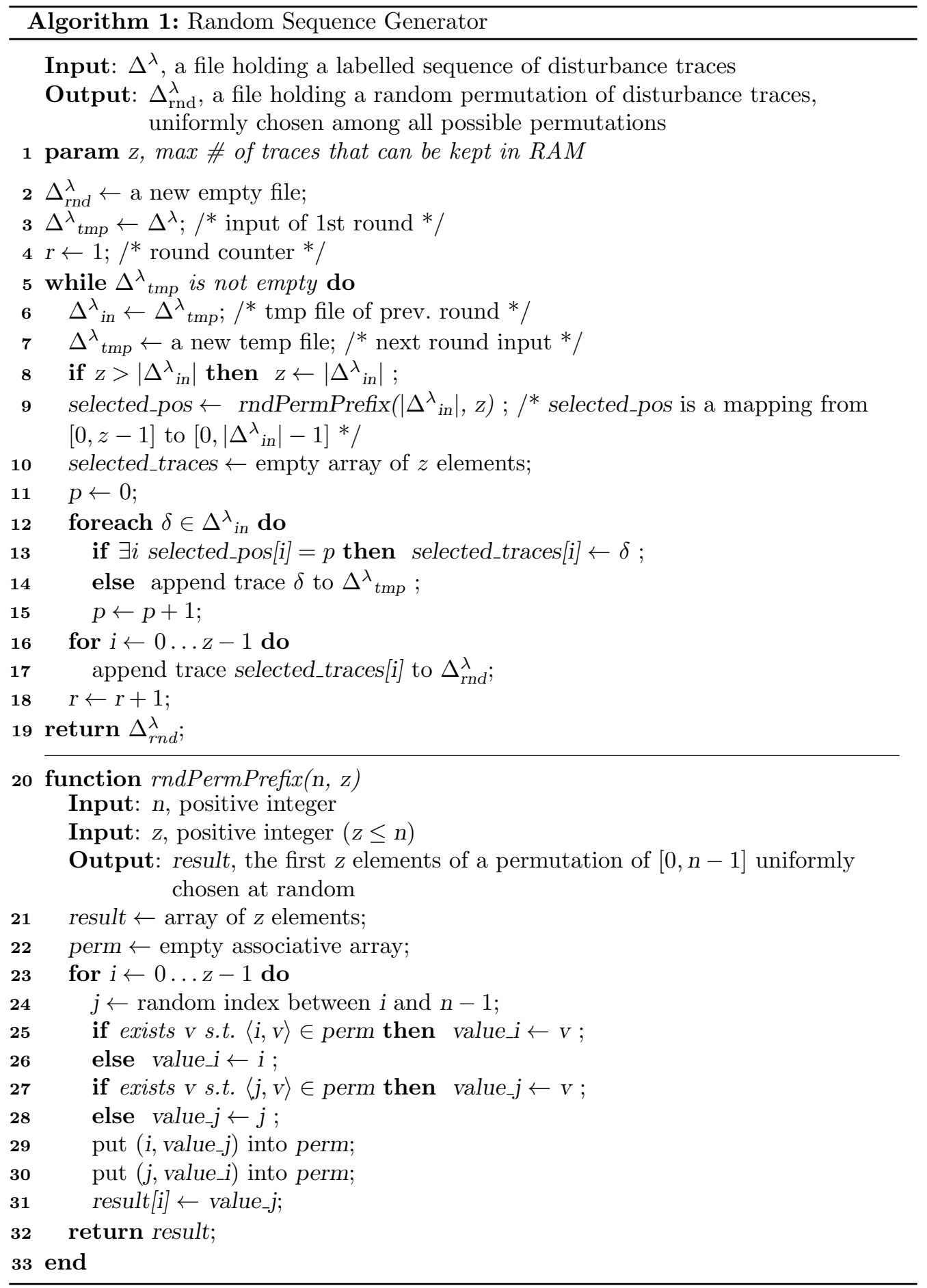




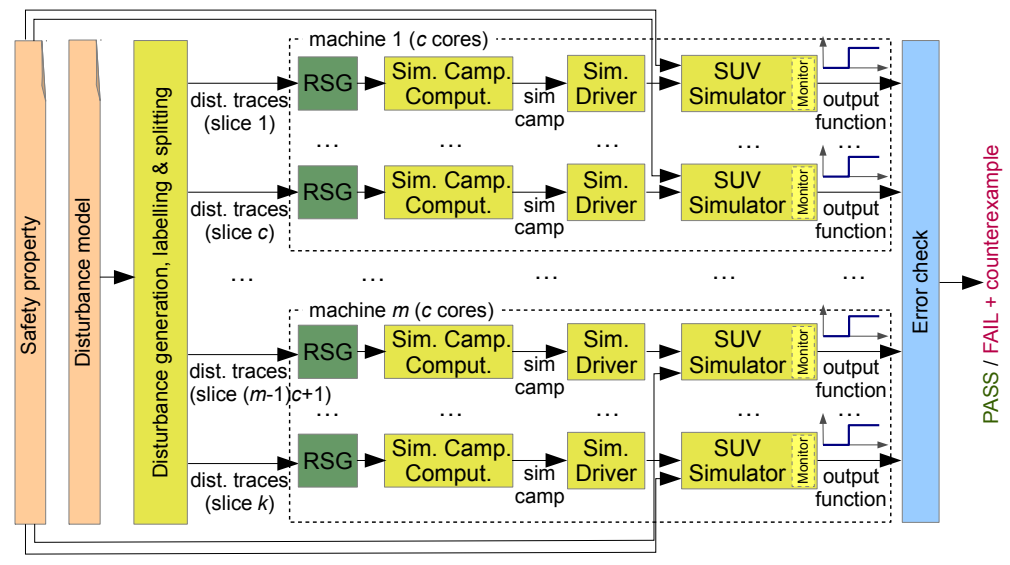

Figure 6: Parallel HILS based rSLFV

the computation as soon as the first $z$ elements (see variable result) have been determined. During its operation, function rndPermPrefix() represents the partially computed permutation as an associative array perm, which, at any step of the function execution, represents permutation $\pi$ of $[0, n-1]$ such that, for each $j \in[0, n-1]$ :

$$
\pi(j)= \begin{cases}v & \text { if }(j, v) \in \text { perm } \\ j & \text { otherwise, i.e., } \nexists v(j, v) \in \text { perm }\end{cases}
$$

The main algorithm then appends the $z$ randomly selected traces (as chosen by function rndPermPrefix ()$)$ to $\Delta_{\text {rnd }}^{\lambda}$ (according to their output positions), and dumps all the others to a temporary file, which becomes the input of the next round. Algorithm 1 terminates in $\lceil n / z\rceil$ rounds.

The following theorem asserts that Algorithm 1, when applied to a sequence of disturbance traces $\Delta^{\lambda}$, produces a uniformly chosen permutation over the set of all permutations. The proof of the theorem is in Section 5.

Theorem 2. Let $\Delta^{\lambda}$ be a file containing $n$ disturbance traces. For any $z \geq 1$, Algorithm 1 stores in file $\Delta_{\text {rnd }}^{\lambda}$ a permutation of the traces in $\Delta^{\lambda}$ extracted with uniform probability among all possible permutations.

The $k$ output randomised slices (computed in parallel by $k$ instances of the Random Sequence Generator algorithm from $k$ input slices) are then given as input to $k$ parallel instances of the simulation campaign computation module described in Section 2.5.2 which compute $k$ highly optimised simulation campaigns (Figure 6). As each simulation campaign verifies the traces in its input slice according to the their order, the introduction of the Random Sequence Generator module effectively enforces a random order in the disturbance trace verification within each slice, satisfying the requirements stated in Theorem 1 in order to compute, during simulation, an upper bound to the $\mathrm{OP}$. 


\section{Proof of Results}

Theorem 1. Let $(h, d, \Delta, \mathcal{H})$ be a System Level Formal Verification (SLFV) problem and $\left(\Delta_{0}, \ldots, \Delta_{k-1}\right)$ be a partition of $\Delta$ into $k \in \mathbb{N}^{+}$disjoint non-empty sets. Let $(\Omega, \mathcal{F}, \operatorname{Pr})$ be a Random Sequence Generator for $\left(\Delta_{0}, \ldots, \Delta_{k-1}\right)$ and $\left(q_{0}, \ldots, q_{k-1}\right)$ a tuple such that $q_{i} \in\left\{0, \ldots,\left|\Delta_{i}\right|\right\}$ for each $0 \leq i<k$. We have:

$$
\widetilde{O P}_{H}\left(\left|\Delta_{0}\right|, \ldots,\left|\Delta_{k-1}\right|, q_{0}, \ldots, q_{k-1}\right) \leq 1-\min \left\{\frac{q_{i}}{\left|\Delta_{i}\right|} \mid 0 \leq i<k\right\}
$$

Proof. If, for all $\delta \in \Delta, \mathcal{H}(\delta)=0$, then the left member of $(1)$ is $\operatorname{Pr}(\emptyset)=0$ and the thesis follows.

Otherwise, let $E$ be a nonempty set containing error traces, that is $E=\{\delta \mid \delta \in$ $\Delta \wedge \mathcal{H}(\delta)=1\} \neq \emptyset . \mathrm{OP}_{\mathcal{H}}\left(\left|\Delta_{0}\right|, \ldots,\left|\Delta_{k-1}\right|, q_{0}, \ldots, q_{k-1}\right)$ can be rewritten as (see Definition 8):

$\operatorname{Pr}\left(\left\{\left(\omega_{0}, \ldots, \omega_{k-1}\right) \mid \forall i \in[0, k-1] \omega_{i} \in \Omega_{i} \wedge \forall i \in[0, k-1] \forall j \in\left[0, q_{i}-1\right] \omega_{i}(j) \notin E\right\}\right)$

because $\left(\Delta_{0}, \ldots, \Delta_{k-1}\right)$ is a partition of $\Delta$.

Consider any $\bar{\delta} \in E$. Probability (2) is less than or equal to

$\operatorname{Pr}\left(\left\{\left(\omega_{0}, \ldots, \omega_{k-1}\right) \mid \forall i \in[0, k-1] \omega_{i} \in \Omega_{i} \wedge \forall i \in[0, k-1] \forall j \in\left[0, q_{i}-1\right] \omega_{i}(j) \neq \bar{\delta}\right\}\right)$

Given that $\bar{\delta}$ belongs to exactly one of $\Delta_{0}, \ldots, \Delta_{k-1}$, say $\Delta_{\bar{i}}$, and given the definition of $\operatorname{Pr}\left(\omega_{0}, \ldots, \omega_{k-1}\right)$ in Definition 7 , expression (3) is equal to:

$$
\left(\prod_{\substack{i=0 \\ i \neq \bar{i}}}^{k-1} \operatorname{Pr}\left(\left\{\omega_{i} \in \Omega_{i}\right\}\right)\right) \times \operatorname{Pr}\left(\left\{\omega_{\bar{i}} \in \Omega_{\bar{i}} \mid \forall j \in\left[0, q_{\bar{i}}-1\right] \omega_{\bar{i}}(j) \neq \bar{\delta}\right\}\right)
$$

which is equal to

$$
\operatorname{Pr}\left(\left\{\omega_{\bar{i}} \in \Omega_{\bar{i}} \mid \forall j \in\left[0, q_{\bar{i}}-1\right] \omega_{\bar{i}}(j) \neq \bar{\delta}\right\}\right)
$$

as, for each $i \neq \bar{i}(0 \leq i<k), \operatorname{Pr}\left(\left\{\omega_{i} \in \Omega_{i}\right\}\right)=1$.

Probability (4) is the probability of picking a permutation $\omega_{\bar{i}}$ of $\Delta_{\bar{i}}$ which does not have $\bar{\delta}$ in the first $q_{\bar{i}}$ positions, and evaluates to $1-\frac{q_{\bar{i}}\left(\left|\Delta_{\bar{i}}\right|-1\right) !}{\left|\Delta_{\bar{i}}\right| !}=1-\frac{q_{\bar{i}}}{\left|\Delta_{\bar{i}}\right|}$ which is less than or equal to $1-\min \left\{\frac{q_{i}}{\left|\Delta_{i}\right|} \mid 0 \leq i<k\right\}$. The thesis follows.

Theorem 2. Let $\Delta^{\lambda}$ be a file containing $n$ disturbance traces. For any $z \geq 1$, Algorithm 1 stores in file $\Delta_{r n d}^{\lambda}$ a permutation of the traces in $\Delta^{\lambda}$ extracted with uniform probability among all possible permutations.

Proof. We prove the following: (i) all traces in the input sequence (file $\Delta^{\lambda}$ ) will occur in the output sequence (file $\Delta_{\text {rnd }}^{\lambda}$ ) exactly once (i.e., the algorithm computes a permutation); (ii) for each $\delta$ occurring in $\Delta^{\lambda}$, the probability that $\delta$ occurs in $\Delta_{\text {rnd }}^{\lambda}$ at any position is $\frac{1}{n}$ (i.e., the computed permutation is uniformly extracted among all possible permutations). 
Point (i) is immediate: at each round, $z$ traces are appended to $\Delta_{\text {rnd }}^{\lambda}$, all the others are appended to $\Delta^{\lambda}$ tmp , which becomes the input of the next round. Also, the algorithm terminates only if the $\Delta^{\lambda}$ tmp produced at the previous round is empty.

To prove point (iii), for any $p, q$ in $[0, n-1]$, we compute the probability that trace $\delta$ having position $p$ in the input sequence (file $\Delta^{\lambda}$ ) will have position $q$ in the output sequence (file $\Delta_{\text {rnd }}^{\lambda}$ ).

We omit to prove that function $\operatorname{rndPermPrefix}(n, z)$ actually computes the first $z$ elements of a permutation of the integer interval $[0, n-1]$ uniformly selected at random, as the function interrupts a well-known swap-based permutation algorithm as soon as the first $z$ elements have been determined.

Given that, at each round $r \geq 1$, the main algorithm selects the $z$ input traces which will have output positions $(r-1) z$ to $\min (n, r z-1), \delta$ is selected only at round $r_{\delta}=\lceil(q+1) / z\rceil$. Thus, the probability that $\delta$, having input position $p$, will have output position $q$ is:

$$
\operatorname{Pr}\left(\left(\bigcap_{r=1}^{r_{\delta}-1} \neg E_{r}\right) \cap O_{r_{\delta}^{\prime}}^{q^{\prime}}\right)
$$

where, for all $r, \neg E_{r}$ denotes the event " $\delta$ is not selected at round $r$ " and $O_{r \delta}^{q^{\prime}}$ denotes the event " $\delta$ is the $q^{\prime}$-th trace selected in round $r_{\delta}$," where $q^{\prime}=\left(q-z\left(r_{\delta}-1\right)\right)$.

By the chain rule, (5) becomes:

$$
\operatorname{Pr}\left(\neg E_{1}\right) \times \cdots \times \operatorname{Pr}\left(\neg E_{r_{\delta}-1} \mid \neg E_{r_{\delta}-2}, \ldots \neg E_{1}\right) \times \operatorname{Pr}\left(O_{r_{\delta}}^{q^{\prime}} \mid \neg E_{r_{\delta}-1}, \ldots \neg E_{1}\right) .
$$

For all $1 \leq r \leq r_{\delta}-1$ :

$$
\operatorname{Pr}\left(\neg E_{r} \mid \neg E_{r-1}, \ldots \neg E_{1}\right)=\frac{n-z(r-1)-1}{n-z(r-1)} \times \cdots \times \frac{n-z(r-1)-z}{n-z(r-1)-(z-1)}
$$

where, for all $0 \leq i<z$, the $i$-th factor is the number of ways we can choose a trace different from $\delta$ out of $n-z(r-1)-i$ (where $n-z(r-1)$ is the number of traces still not selected at the beginning of round $r$ ). The expression simplifies to:

$$
\operatorname{Pr}\left(\neg E_{r} \mid \neg E_{r-1}, \ldots \neg E_{1}\right)=\frac{n-z r}{n-z(r-1)}
$$

and the product $\operatorname{Pr}\left(\neg E_{1}\right) \times \cdots \times \operatorname{Pr}\left(\neg E_{r_{\delta}-1} \mid \neg E_{r_{\delta}-2}, \ldots \neg E_{1}\right)$ is:

$$
\operatorname{Pr}\left(\neg E_{1}\right) \times \cdots \times \operatorname{Pr}\left(\neg E_{r_{\delta}-1} \mid \neg E_{r_{\delta}-2}, \ldots \neg E_{1}\right)=\prod_{r=1}^{r_{\delta}-1} \frac{n-z r}{n-z(r-1)}=\frac{n-z\left(r_{\delta}-1\right)}{n} .
$$

As for $\operatorname{Pr}\left(O_{r_{\delta}}^{q^{\prime}} \mid \neg E_{r_{\delta}-1}, \ldots \neg E_{1}\right)$, i.e., the probability that $\delta$ is the $q^{\prime}$-th selected trace in round $r_{\delta}$ provided that it has not been selected in previous rounds, it can be computed as:

$$
\frac{n-z\left(r_{\delta}-1\right)-1}{n-z\left(r_{\delta}-1\right)} \times \cdots \times \frac{n-z\left(r_{\delta}-1\right)-\left(q^{\prime}-1\right)}{n-z\left(r_{\delta}-1\right)-\left(q^{\prime}-2\right)} \times \frac{1}{n-z\left(r_{\delta}-1\right)-\left(q^{\prime}-1\right)}=\frac{1}{n-z\left(r_{\delta}-1\right)}
$$




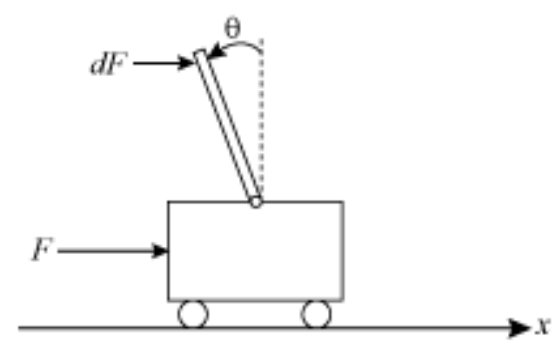

Figure 7: Inverted Pendulum on a Cart (IPC) case study (from mathworks.com).

where, for all $0 \leq i<q^{\prime}-1$, the $i$-th factor is the number of ways we can choose a trace different from $\delta$ out of $n-z\left(r_{\delta}-1\right)-i$ (where $n-z\left(r_{\delta}-1\right)$ is the number of traces still not selected at the beginning of round $\left.r_{\delta}\right)$. The last factor is the probability of selecting $\delta$ (still unselected) out of $n-z\left(r_{\delta}-1\right)-\left(q^{\prime}-1\right)$ traces.

By (7) and (8), probability (6) evaluates to $\frac{1}{n}$, which is independent of $p$ and $q$.

\section{Experimental Results}

In this section we evaluate the effectiveness of our Random Exhaustive SLFV (rSLFV) approach as follows. First, we evaluate the overhead due to the randomisation of disturbance traces needed to enable computation of Omission Probability (OP), by comparing our [rSLFV approach with the Deterministic SLFV (dSLFV] approach of [8]. Second, we evaluate the behaviour of the coverage and the $\mathrm{OP}$ bound with respect to simulation time. Third, we evaluate speed-up and efficiency of our parallel approach.

\subsection{Experimental Setting}

In this section we describe the case studies and the computational infrastructure we used in our experiments.

\subsubsection{Case Studies}

We experiment with two case studies, using two models included in the Simulink distribution, namely the Inverted Pendulum on a Cart (IPC) and the Fuel Control System (FCS).

6.1.1.1. Inverted Pendulum on a Cart (IPC). The IPC is a control loop system where the controlled system is an inverted pendulum installed on a cart (see Figure 7). The controller (actually a control software) senses the angular position $\theta$ of the pendulum, and computes the force $F$ to be applied to the cart to move it left or right along the $x$ axis. The goal is to keep the pendulum in its upright (vertical) unstable position. The physical constraint between the cart and the pendulum gives that both the cart and the pendulum have one degree of freedom each ( $x$ and $\theta$, respectively).

The controlled system consists of the cart and the pendulum, whereas the controller consists of the control software computing $F$ from the plant outputs $(x$ and $\theta)$. Accordingly, our overall System Under Verification (SUV) model consists of the controlled 


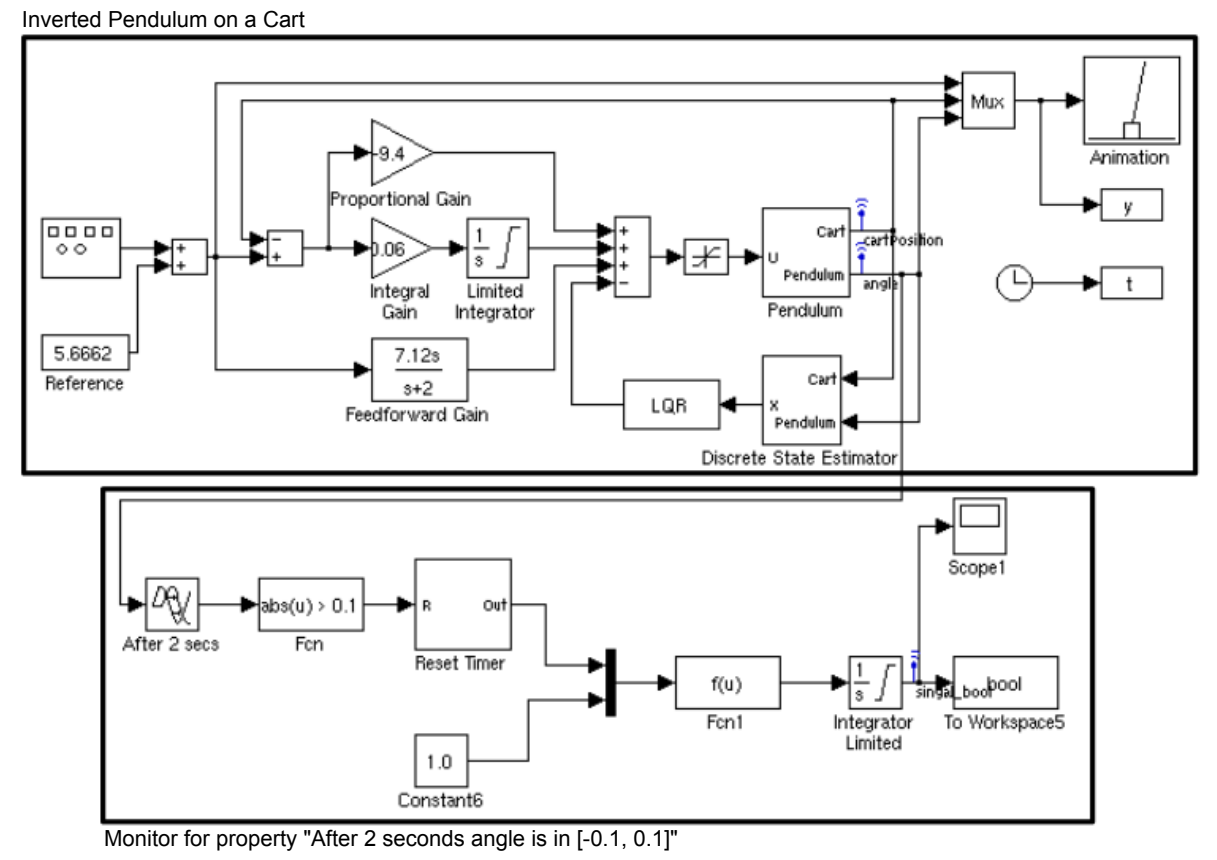

Figure 8: Simulink block diagram for Inverted Pendulum on a Cart (from mathworks.com) with an embedded property monitor.

system and the controller, whose Simulink block diagram is shown in the upper box of Figure 8

The system level property that we verify is that after 2 secs the pendulum is in upright position, i.e., angle $\theta$ is always between $[-0.1,0.1]$. The monitor checking for this property is shown in the lower box of Figure 8

We introduce disturbances by injecting irregularities in the cart rail. We model such irregularities with a modification on the cart weight $m$ with respect to its nominal value of $0.455 \mathrm{~kg}$. For this, we define three disturbances representing normal rail operation $(m=0.455 \mathrm{~kg})$, abnormal rail operation $(m=1.455 \mathrm{~kg})$, and stressed rail operation $(m=2.455 \mathrm{~kg})$.

We consider two disturbance models for the $\llbracket \mathcal{D}_{\text {IPC }}^{1}$ and $\mathcal{D}_{\text {IPC }}^{2}$. Model $\mathcal{D}_{\text {IPC }}^{1}$ has a horizon of $h=90$ and defines 3208276 disturbance traces. Model $\mathcal{D}_{\mathrm{IPC}}^{2}$ is defined extending $\mathcal{D}_{\text {IPC }}^{1}$ with more complex operational scenarios and defines 35641501 disturbance traces over a horizon of $h=200$. For both models we set $\tau$ (quantum between disturbances) to 0.1 second. A detailed description of $\mathcal{D}_{\mathrm{IPC}}^{1}$ and $\mathcal{D}_{\mathrm{IPC}}^{2}$ is not relevant for the evaluation of our experiments below. We only observe that, in our setting, the complexity of answering a System Level Formal Verification (SLFV) problem primarily depends on the number of disturbance traces to be simulated. Thus, the worst case for our approach is when all disturbance traces have to be simulated, i.e., when the answer to the SLFV problem is PASS. Indeed, both $\mathcal{D}_{\mathrm{IPC}}^{1}$ and $\mathcal{D}_{\mathrm{IPC}}^{2}$ satisfy this requirement. 
6.1.1.2. Fuel Control System (FCS). The FCS is a controller for a fault tolerant gasoline engine, which has also been used as a case study in [15, 16, 17, 18, 8, 12,).

The FCS has four sensors: throttle angle, speed, EGO (measuring the residual oxygen present in the exhaust gas) and MAP (manifold absolute pressure). The goal of the control system is to maintain the air-fuel ratio (the ratio between the air mass flow rate pumped from the intake manifold and the fuel mass flow rate injected at the valves) close to the stoichiometric ratio of 14.6 , which represents a good compromise between power, fuel economy, and emissions.

From the sensor measurements, the FCS estimates the mixture ratio and provides feedback to the closed-loop control, yielding an increase or a decrease of the fuel rate.

The FCS sensors are subject to faults (disturbances), and the whole control system can tolerate single sensor faults. In particular, if a sensor fault is detected, the FCS changes its control law by operating the engine with a higher fuel rate to compensate. In case two or more sensors fail, the FCS shuts down the engine, as the air-fuel ratio cannot be controlled.

The control logic of the FCS is implemented by six automata, each one with a number of states ranging from two to five. The signal flow is further subdivided into three subsystems, which exhibit several different Simulink block types, involving arithmetic, lookup tables, integrators, filters and interpolation [19] (see [20] for more details).

We verify one of the system level specifications for such a model, namely: the fuel_air model variable is never 0 for more than one second. Accordingly, our SUV consists of the Simulink [FCS model along with a monitor for the property under verification (such a model is shown as Figure 9p.

We consider two disturbance models for the $\left[\mathrm{FCS}, \mathcal{D}_{\mathrm{FCS}}^{1}\right.$ and $\mathcal{D}_{\mathrm{FCS}}^{2}$. Model $\mathcal{D}_{\mathrm{FCS}}^{1}$ has a horizon of $h=100$ and defines 4023955 disturbance traces. Model $\mathcal{D}_{\text {FCS }}^{2}$ is defined extending $\mathcal{D}_{\text {FCS }}^{1}$ with more complex operational scenarios and defines 12948712 disturbance traces over a horizon of $h=200$. For both models we set $\tau$ (quantum between disturbances) to 1 second. A detailed description of $\mathcal{D}_{\mathrm{FCS}}^{1}$ and $\mathcal{D}_{\mathrm{FCS}}^{2}$ is not relevant for the evaluation of our experiments below, and can be found in 8 , 21. We only observe that, as it happens with our disturbance models for the IPC, for all disturbance traces entailed by $\mathcal{D}_{\mathrm{FCS}}^{1}$ and $\mathcal{D}_{\mathrm{FCS}}^{2}$, the property to be verified is satisfied. This yields the worst scenario to answer our $[\mathrm{SLFV}]$ problem, as all traces need to be simulated.

\subsubsection{Computational Infrastructure}

We ran experiments on multiple Linux PCs, each one equipped with 2 Intel Xeon 3.0 $\mathrm{GHz}$ CPUs with 4 cores each and 8 GB RAM. We executed 8 processes (optimisation and simulation) in parallel (one per available core) on each machine.

As, in a multi-core setting, the local disk may quickly become a performance bottleneck if heavily used by multiple processes, we have replaced it with 8 RAM disks of 500 $\mathrm{MB}$ each per machine, in order to store simulation states. Accordingly, we have used the multi-core version of the dSLFV optimiser of [8] as presented in [12]. Given that, in our case studies, the size of the simulation state files is of about $150-300 \mathrm{~KB}$, this experimental setting allowed our optimiser to count on the possibility, for each simulator, to keep at most 1800 states simultaneously stored. 


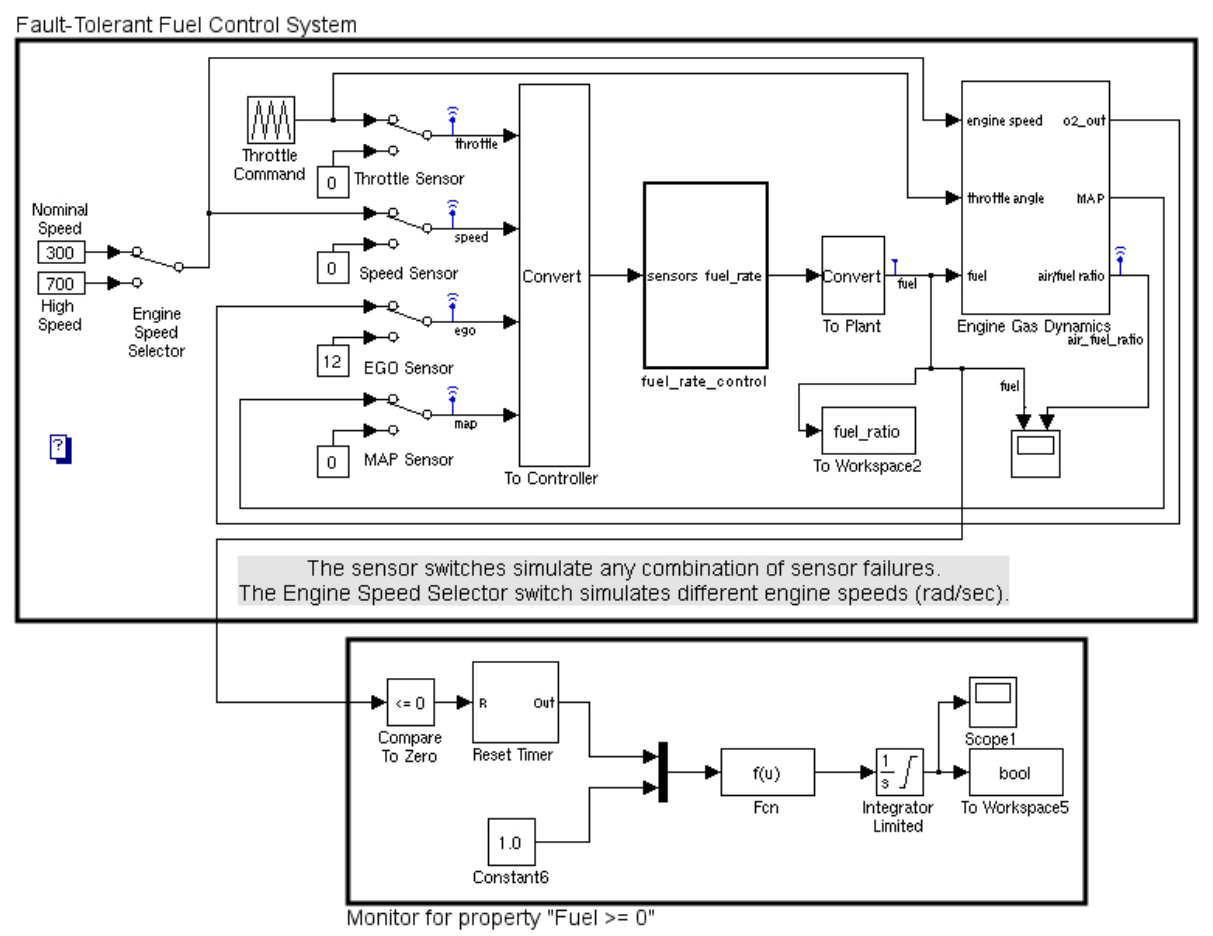

Figure 9: Simulink/Stateflow representation of the Fuel Control System (from mathworks.com) with an embedded property monitor.

\subsection{Disturbance Trace Generation and Splitting}

Along the lines of $[8$, we use CMurphi to generate the set of labelled admissible disturbance traces entailed by the disturbance model given as input.

Table 1 shows, for each of the four disturbance models we consider (two for IPC and two for FCS) the number of entailed disturbance traces (column \#traces), the time needed by CMurphi to generate them (column gen. time) and the size of the file computed by our generator to store them (column file size).

We then split the generated sequences of disturbance traces into $k$ slices, with $k=$ $128,256,512$ to enable parallel computation on, respectively, 16, 32, 64 (8-core) machines. Splitting always takes negligible time.

\subsection{Computation of Optimised Simulation Campaigns}

Table 2 shows, for each of the disturbance models we consider, the time needed by our Random Sequence Generator module to enable anytime $\mathrm{OP}$ computation during the verification process (rSLFV) and the time needed to compute optimised simulation campaigns with (rSLFV) and without dSLFV trace order randomisation.

Column \#mach gives the number of machines we used in parallel. Column \#slices gives the overall number of slices in which the sequence of admissible disturbance traces has been partitioned (one per available core, i.e., 8 slices per machine). Column \#traces 


\begin{tabular}{ccccc}
\hline SUV & dist. model & \#traces & gen. time & file size \\
\hline IPC & $\mathcal{D}_{\text {IPC }}^{1}$ & 3208276 & $0: 9: 58$ & $4.6 \mathrm{~GB}$ \\
IIPC & $\mathcal{D}_{\text {IPC }}^{2}$ & 35641501 & $7: 28: 24$ & $107 \mathrm{~GB}$ \\
\hline FCS & $\mathcal{D}_{\mathrm{FCS}}^{1}$ & 4023955 & $0: 28: 39$ & $3.5 \mathrm{~GB}$ \\
FCS & $\mathcal{D}_{\mathrm{FCS}}^{2}$ & 12948712 & $4: 45: 47$ & $39 \mathrm{~GB}$ \\
\hline
\end{tabular}

Table 1: Disturbance trace generation.

per slice shows the number of traces in any single slice (except the last slice, which has fewer traces when the overall number of traces is not a multiple of \#slices). Column group $r S L F V$ shows the maximum overall time to compute the simulation campaign starting from a slice. This time is split into two parts: the time needed to execute the Random Sequence Generator module (column $[R S G$ ) and the time needed to compute the simulation campaign starting from the randomised slice (column sim. camp. comp.). Column $d S L F V$ shows the maximum time to compute the simulation campaign starting from a slice when no RSG is performed, thus no anytime OP computation is enabled. Column rSLFV overhead shows the difference between the rSLFV] and dSLFV times.

It can be observed that our parallel approach to computation of optimised randomised simulation campaigns is able to effectively exploit parallelism in order to handle disturbance models entailing tens of millions of operational scenarios.

Also, Table 2 shows that the random sequence generation phase makes the rSLFV] simulation campaign computation process longer than that for dSLFV (i.e., overhead is positive). The difference is, however, negligible with respect to the whole verification time, which takes many hours, even for the massively parallel simulation of operational scenarios entailed by our smallest disturbance models, i.e., $\mathcal{D}_{\text {IPC }}^{1}$ for the $\amalg$ C and $\mathcal{D}_{\text {FCS }}^{1}$ for the FCS (see Section 6.4).

\subsection{Execution of the Simulation Campaigns}

Table 3 shows the execution time of the simulation campaigns generated by dSLFV and rSLFV

As the exhaustive simulation of the traces generated by disturbance models $\mathcal{D}_{\text {IPC }}^{2}$, $\mathcal{D}_{\text {FCS }}^{2}$ (entailing, respectively, 35641501 and 12948712 disturbance traces) would require a prohibitively long time, from now on we restrict ourselves to the simulation of the traces generated by $\mathcal{D}_{\text {IPC }}^{1}$ and $\mathcal{D}_{\text {FCS }}^{1}$ (which entail, respectively, 3208276 and 4023955 disturbance traces).

By enabling the computation of the $\mathrm{OP}$ during the simulation activity we have a quite significant increase of simulation time. However, such an overhead can be drastically reduced, or even neutralised, by using more parallel processes (higher values for $k=$ \#slices). This behaviour is due to the fact that the rSLFV optimiser needs to compute a simulation campaign under the restriction that the number of states that the simulator can keep simultaneously stored is limited (to fit within the $500 \mathrm{MB}$ of disk space available to each simulator instance). For high values of $k=\#$ slices (e.g., $k=512$ ), this is not a big obstacle. On the other hand, for low values of $k$, the number of traces in each slice is higher and they share shorter common prefixes on average. Hence, a fully-optimised 


\begin{tabular}{|c|c|c|c|c|c|c|c|}
\hline \#mach. & \#slices & $\begin{array}{l}\text { \#traces } \\
\text { per slice }\end{array}$ & RSG & $\begin{array}{c}\text { rSLFV } \\
\text { sim.camp.comp. }\end{array}$ & total & $\begin{array}{c}\text { dSLFV } \\
\text { sim.camp.comp. }\end{array}$ & $\begin{array}{l}\text { rSLFV } \\
\text { overhead }\end{array}$ \\
\hline 1 & 8 & 401035 & $0: 0: 21$ & $1: 8: 52$ & $1: 9: 13$ & $0: 8: 53$ & 1:0:20 \\
\hline 2 & 16 & 200518 & $0: 0: 11$ & $0: 11: 57$ & $0: 12: 9$ & $0: 4: 36$ & $0: 7: 32$ \\
\hline 4 & 32 & 100259 & $0: 0: 5$ & $0: 6: 33$ & $0: 6: 38$ & $0: 2: 23$ & $0: 4: 14$ \\
\hline 8 & 64 & 50130 & $0: 0: 3$ & $0: 2: 50$ & $0: 2: 53$ & $0: 1: 14$ & 0:1:39 \\
\hline 16 & 128 & 25065 & $0: 0: 2$ & $0: 1: 23$ & $0: 1: 26$ & $0: 0: 38$ & $0: 0: 47$ \\
\hline 32 & 256 & 12533 & 0:0:1 & $0: 1: 19$ & $0: 1: 21$ & $0: 0: 20$ & 0:1:1 \\
\hline 64 & 512 & 6267 & 0:0:1 & $0: 0: 21$ & $0: 0: 23$ & $0: 0: 11$ & 0:0:12 \\
\hline
\end{tabular}

(a) Inverted Pendulum on a Cart (IPC), disturbance model $\mathcal{D}_{\text {IPC }}^{1}: 3208276$ traces with horizon $h=90$

\begin{tabular}{|c|c|c|c|c|c|c|c|}
\hline \#mach. & \#slices & $\begin{array}{l}\text { \#traces } \\
\text { per slice }\end{array}$ & RSG & $\begin{array}{c}\text { rSLFV } \\
\text { sim.camp.comp. }\end{array}$ & total & \begin{tabular}{c|} 
dSLFV \\
sim.camp.comp.
\end{tabular} & $\begin{array}{l}\text { rSLFV } \\
\text { overhead }\end{array}$ \\
\hline 16 & 128 & 278450 & $0: 1: 40$ & $2: 41: 28$ & $2: 43: 8$ & $0: 35: 0$ & $2: 8: 8$ \\
\hline 32 & 256 & 139225 & $0: 1: 6$ & 1:9:12 & $1: 10: 18$ & $0: 17: 40$ & 0:52:38 \\
\hline 64 & 512 & 69613 & 0:0:34 & $0: 21: 26$ & 0:22:0 & $0: 8: 57$ & 0:13:3 \\
\hline
\end{tabular}

(b) Inverted Pendulum on a Cart (IPC), disturbance model $\mathcal{D}_{\text {IPC }}^{2}: 35641501$ traces with horizon $h=200$

\begin{tabular}{|c|c|c|c|c|c|c|c|}
\hline \#mach. & \#slices & $\begin{array}{l}\text { \#traces } \\
\text { per slice }\end{array}$ & RSG & $\begin{array}{c}\text { rSLFV } \\
\text { sim.camp.comp. }\end{array}$ & total & $\begin{array}{c}\text { dSLFV } \\
\text { sim.camp.comp. }\end{array}$ & \begin{tabular}{|c|} 
rSLFV \\
overhead
\end{tabular} \\
\hline 1 & 8 & 502995 & |0:1:12 & $0: 4: 52$ & $0: 6: 4$ & $0: 5: 27$ & 0:0:37 \\
\hline 2 & 16 & 251498 & $0: 0: 24$ & $0: 2: 34$ & $0: 2: 58$ & $0: 2: 8$ & 0:0:50 \\
\hline 4 & 32 & 125749 & $0: 0: 15$ & $0: 2: 24$ & $0: 2: 39$ & $0: 0: 57$ & $0: 1: 42$ \\
\hline 8 & 64 & 62875 & $0: 0: 8$ & $0: 1: 20$ & $0: 1: 28$ & $0: 0: 29$ & 0:0:59 \\
\hline 16 & 128 & 31438 & $0: 0: 7$ & $0: 1: 19$ & $0: 1: 26$ & $0: 0: 17$ & $0: 1: 9$ \\
\hline 32 & 256 & 15719 & $0: 0: 7$ & 0:0:32 & $0: 0: 39$ & $0: 0: 8$ & 0:0:31 \\
\hline 64 & 512 & 7860 & $0: 0: 6$ & $0: 0: 5$ & $0: 0: 11$ & $0: 0: 4$ & $0: 0: 7$ \\
\hline
\end{tabular}

(c) Fuel Control System ( $(\underline{\text { FCS }})$, disturbance model $\mathcal{D}_{\text {FCS }}^{1}: 4023955$ traces with horizon $h=100$

\begin{tabular}{|c|c|c|c|c|c|c|c|}
\hline \multirow[t]{2}{*}{ \#mach. } & \multirow[t]{2}{*}{ \#slices } & \multirow{2}{*}{$\begin{array}{l}\text { \#traces } \\
\text { per slice }\end{array}$} & \multicolumn{3}{|c|}{ rSLFV } & \multirow{2}{*}{\begin{tabular}{|c|} 
dSLFV \\
sim.camp.comp.
\end{tabular}} & \multirow{2}{*}{\begin{tabular}{|c|} 
rSLFV \\
overhead
\end{tabular}} \\
\hline & & & RSG & sim.camp.comp. & total & & \\
\hline 16 & 128 & 101162 & 0:0:45 & $0: 20: 36$ & $0: 21: 21$ & $0: 8: 18$ & $0: 13: 3$ \\
\hline 32 & 256 & & 0:0:24 & $0: 15: 35$ & $0: 1$ & $0: 4: 31$ & $0: 11: 28$ \\
\hline 64 & 512 & 25291 & 0:0:14 & $0: 6: 43$ & $0: 6: 57$ & $0: 2: 4$ & $0: 4: 53$ \\
\hline
\end{tabular}

(d) Fuel Control System ( $(\underline{\text { FCS }})$, disturbance model $\mathcal{D}_{\text {FCS }}^{2}: 12948712$ traces with horizon $h=200$

Table 2: Overhead of enabling OP (rSLFV with respect to dSLFV in the computation of simulation campaigns (time in h:m:s). 


\begin{tabular}{cccccc}
\hline \#mach. & \#slices & min & max & avg & approach \\
\hline \multirow{2}{*}{16} & \multirow{2}{*}{128} & $2: 14: 2$ & $4: 10: 52$ & $3: 44: 52$ & dSLFV \\
& & $25: 14: 28$ & $85: 10: 36$ & $58: 9: 43$ & rSLFV \\
\cline { 3 - 6 } & & $\mathbf{2 3 : 0 : 2 6}$ & $\mathbf{8 0 : 5 9 : 4 4}$ & $\mathbf{5 4 : 2 4 : 5 1}$ & overhead \\
\hline \multirow{2}{*}{25} & $1: 6: 49$ & $2: 5: 40$ & $1: 50: 28$ & dSLFV \\
& & $4: 26: 16$ & $23: 59: 0$ & $13: 0: 24$ & rSLFV \\
\cline { 3 - 6 } & \multirow{3}{*}{$5: \mathbf{1 9 : 2 7}$} & $\mathbf{2 1 : 5 3 : 2 0}$ & $\mathbf{1 1 : 9 : 5 6}$ & overhead \\
\hline \multirow{2}{*}{54} & $0: 30: 30$ & $1: 3: 10$ & $0: 53: 45$ & dSLFV \\
& & $0: 59: 26$ & $1: 51: 10$ & $1: 43: 12$ & rSLFV \\
\cline { 3 - 6 } & & $\mathbf{0 : 2 8 : 5 6}$ & $\mathbf{0 : 4 8 : 0}$ & $\mathbf{0 : 4 9 : 2 7}$ & overhead \\
\hline
\end{tabular}

(a) Inverted Pendulum on a Cart (IPC), disturbance model $\mathcal{D}_{\text {IPC }}^{1}$

\begin{tabular}{cccccc}
\hline \#mach. & \#slices & min & max & avg & approach \\
\hline \multirow{2}{*}{16} & \multirow{2}{*}{128} & $70: 6: 4$ & $100: 17: 53$ & $87: 49: 56$ & dSLFV \\
& & $216: 42: 13$ & $348: 51: 47$ & $308: 46: 18$ & rSLFV \\
\cline { 3 - 6 } & & $\mathbf{1 4 6 : 3 6 : 9}$ & $\mathbf{2 4 8 : 3 3 : 5 4}$ & $\mathbf{2 2 0 : 5 6 : 2 2}$ & overhead \\
\hline \multirow{2}{*}{25} & $44: 0: 27$ & $57: 57: 27$ & $48: 34: 6$ & dSLFV \\
& & $63: 53: 54$ & $136: 18: 14$ & $108: 14: 19$ & rSLFV \\
\cline { 3 - 6 } & \multirow{2}{*}{512} & $18: 53: 27$ & $\mathbf{7 8 : 2 0 : 4 7}$ & $\mathbf{5 9 : 4 0 : 1 3}$ & overhead \\
\hline \multirow{2}{*}{64} & & $22: 9: 19$ & $29: 23: 33$ & $26: 43: 31$ & rSLFV \\
\cline { 2 - 6 } & & $\mathbf{3 : 3 6 : 4 3}$ & $\mathbf{2 : 3 4 : 2 9}$ & $\mathbf{3 : 4 1 : 1 2}$ & overhead \\
\hline
\end{tabular}

(b) Fuel Control System (

Table 3: Parallel execution of simulation campaigns by dSLFV and rSLFV (time in h:m:s). 
random order execution of them would need a too high number of simulation states to be simultaneously kept stored. As a consequence, the optimiser is forced to post free commands for many simulation states which would be needed again in yet-to-besimulated traces. Such traces will then be simulated from the simulator initial state, thus yielding performance degradation.

\subsection{Overall Verification Time and Scalability}

In this section we evaluate the overall impact of enabling anytime $\mathrm{OP}$ computation and the scalability of our parallel approach to SLFV

Table 4 shows the overall time needed to carry out our SLFV tasks (IPC with disturbance model $\mathcal{D}_{\text {IPC }}^{1}$ and FCS with disturbance model $\mathcal{D}_{\mathrm{FCS}}^{1}$ ) with $k$ parallel processes, for the values of $k$ already used in the previous tables.

In particular, column gen. $\&$ comp. sim. camp. reports the sum of the disturbance trace generation and splitting time, parallel randomisation (only for rSLFV) and parallel simulation campaign computation time (from Table 2). Column simulation reports the parallel simulation time when using $k$ parallel processes (i.e., the maximum simulation time over all the $k=$ \#slices slices as in column max of Table 33. Column overall reports the overall time to carry out each dSLFV and each rSLFV task, as the sum of the previous two columns.

We observe that our approach takes negligible time to generate optimised simulation campaigns with respect to the time needed to actually simulate them (e.g., minutes vs. hours).

\subsubsection{Estimation of Sequential Verification Time}

In order to evaluate the scalability of our parallel approach to SLFV, Table 4 also reports (in the first two rows of each sub-table), the overall dSLFV and rSLFV times when using only one parallel process (sequential time). Unfortunately, as for our case studies a sequential simulation would be prohibitively long, we have estimated the sequential simulation time to carry out both [SLFV and rSLFV as follows.

Let $t_{k}^{\text {avg }}$ be the average time to simulate a slice where $k=$ \#slices parallel processes are used (row \#slices $=k$, column avg, for either dSLFV or rSLFV). For any value of $k$, the sequential simulation time could be estimated as $k \times t_{k}^{\text {avg }}$. As this value changes a little bit for different values of $k$, Table 4 estimates sequential simulation time as $\min \left\{128 t_{128}^{\text {avg }}, 256 t_{256}^{\text {avg }}, 512 t_{512}^{\text {avg }}\right\}$. Such huge values (weeks of computation) make clear that estimation is the only viable way to compute the simulation sequential times. Note that in our computation we are slightly overestimating the sequential time, since we are assuming that some traces of each slice must be simulated from the initial state. In an actual 1-process execution of a simulation campaign, the optimiser may exploit stored simulator states to avoid simulation of such traces from the initial state. As the time to simulate a single trace is of a few seconds and the simulator can keep only a limited number of stored states, this is negligible with respect to the value of the sequential simulation time.

\subsubsection{Speedup and Efficiency}

Sequential simulation time for both dSLFV and rSLFV is used in Table 4 to compute the speedup and the efficiency of our parallel approach to SLFV as typically done in 
the evaluation of parallel algorithms. In particular, for each $k=\#$ slices, column speedup shows the ratio $t_{1} / t_{k}$, where $t_{1}$ is the estimated overall sequential verification time and $t_{k}$ is the overall verification time when $k$ parallel processes are used. Column efficiency is computed by dividing the speedup by the number of parallel processes $k=\#$ slices.

Table 4 also shows the overhead (see bold values) due to randomisation of the verification task (which is the price to pay in order to enable anytime computation of $\mathrm{OP}$ ), both in terms of overall verification time increase and in terms of reduction of speedup and efficiency. We observe that such an overhead is significant, but it can be drastically reduced by increasing the number $k$ of parallel processes.

\subsection{Omission Probability}

Figures 10a and 10c show how our upper bound to the $\mathrm{OP}$ decreases as a function of the coverage (i.e., the ratio of admissible traces simulated) during the parallel execution of the $k$ simulation campaigns $\left[\right.$ IPC with disturbance model $\mathcal{D}_{\text {IPC }}^{1}$ and $\overline{\text { FCS }}$ with disturbance model $\mathcal{D}_{\text {FCS }}^{1}$ ), for $k=128,256,512$. It can be observed that our $\overline{\mathrm{OP}}$ bound is always very close to the ratio of yet-to-be-simulated traces (curves named "100\%-coverage", i.e., $100 \%$ minus coverage), which is the best one can do (using only one parallel process) without any assumption on the number of error traces.

\subsection{Completion Time Estimation}

Figures $10 \mathrm{~b}$ and $10 \mathrm{~d}$ show that $\mathrm{OP}$ bound, computed during the parallel execution of the simulation campaigns (IPC) with disturbance model $\mathcal{D}_{\text {IPC }}^{1}$ and FCS with disturbance model $\mathcal{D}_{\mathrm{FCS}}^{1}$ ), decreases nearly linearly in time. The same happens with the coverage, which can thus be used as a reliable estimator for the completion time of the whole verification process.

Figure 11 shows the error percentage (on the true completion time) made by a completion time estimation based on the coverage. For each value $x$ of the coverage, the error is computed as $\left(\left(t_{x} / x\right)-t_{c}\right) / t_{c}$ where $t_{x}$ is the time elapsed to reach coverage $x$ and $t_{c}$ is the true completion time. It can be observed that such a completion time estimation becomes accurate quickly (e.g., when the coverage is $\geq 30 \%$, the error is within $30 \%$ ).

\section{Related Work}

A parallel exhaustive Hardware In the Loop Simulation based hybrid system model checking similar to the one described in this work is presented in [8]. The main differences of the present work with respect to [8] are the following. (i) Our simulation campaign optimiser and the one in 8 ] both take as input the admissible disturbance traces (simulation scenarios). However, the simulation campaigns computed in 8$]$ schedule scenarios according to their order, whereas in this work we introduce an intermediate step which enables simulation of all scenarios, exactly once, in a uniform random order. (ii) During the verification process, the approach in [8] only outputs the attained coverage, whereas, in this work also the attained Omission Probability ( $(\mathrm{OP})$ is computed, by exploiting the randomisation of the order with which scenarios are scheduled.

The work in [9] considers a finite state (digital hardware verification) setting and presents an algorithm to estimate the coverage achieved during SAT based bounded 


\begin{tabular}{|c|c|c|c|c|c|c|c|}
\hline \#mach. & Fslices & $\begin{array}{l}\text { gen. \& } \\
\text { comp. sim. } \\
\text { camp. }\end{array}$ & simulation & overall & speedup & efficiency & approach \\
\hline \multirow[t]{2}{*}{1} & 1 & $\begin{array}{c}0: 56: 11 \\
26: 59: 57\end{array}$ & $\begin{array}{c}458: 40: 0^{(*)} \\
880: 38: 24^{(*)}\end{array}$ & $\begin{array}{l}\text { 459:36:11(*) } \\
907: 38: 21^{(*)}\end{array}$ & $\begin{array}{l}1.00 \times \\
1.00 \times\end{array}$ & $\begin{array}{l}100.00 \% \\
100.00 \%\end{array}$ & $\begin{array}{l}\text { dSLFV } \\
\text { rSLFV }\end{array}$ \\
\hline & & & & $+97.48 \%^{(*)}$ & $+0.00 \%$ & $+\mathbf{0 . 0 0} \%$ & overhead \\
\hline \multirow[t]{2}{*}{16} & 128 & $\begin{array}{l}0: 10: 36 \\
0: 11: 24\end{array}$ & $\begin{array}{c}4: 10: 52 \\
85: 10: 36\end{array}$ & $\begin{array}{l}4: 21: 28 \\
85: 22: 0\end{array}$ & $\begin{array}{c}105.46 \times \\
10.63 \times\end{array}$ & $\begin{array}{l}82.39 \% \\
8.30 \%\end{array}$ & $\begin{array}{l}\text { dSLFV } \\
\text { rSLFV }\end{array}$ \\
\hline & & & & $\mid+1858.83 \%$ & $+89.92 \%$ & $+74.09 \%$ & overhead \\
\hline \multirow[t]{2}{*}{32} & 256 & $\begin{array}{l}0: 10: 18 \\
0: 11: 19\end{array}$ & $\begin{array}{c}2: 5: 40 \\
23: 59: 0\end{array}$ & $\begin{array}{c}2: 15: 58 \\
24: 10: 19\end{array}$ & $\begin{array}{c}202.80 \times \\
37.55 \times\end{array}$ & $\begin{array}{l}79.22 \% \\
14.67 \%\end{array}$ & $\begin{array}{l}\text { dSLFV } \\
\text { rSLFV }\end{array}$ \\
\hline & & & & $+966.57 \%$ & $\mid+81.48 \%$ & $+\mathbf{6 4 . 5 5} \%$ & overhead \\
\hline \multirow[t]{2}{*}{64} & 512 & $\begin{array}{c}0: 10: 9 \\
0: 10: 21\end{array}$ & $\begin{array}{c}1: 3: 10 \\
1: 51: 10\end{array}$ & $\begin{array}{l}1: 13: 19 \\
2: 1: 31\end{array}$ & $\begin{array}{l}376.04 \times \\
448.13 \times\end{array}$ & $\begin{array}{l}73.45 \% \\
87.53 \%\end{array}$ & $\begin{array}{l}\text { dSLFV } \\
\text { rSLFV }\end{array}$ \\
\hline & & & & $+65.71 \%$ & $-19.17 \%$ & $-14.08 \%$ & overhead \\
\hline
\end{tabular}

(a) Inverted Pendulum on a Cart (IPC), disturbance model $\mathcal{D}_{\text {IPC }}^{1}$

\begin{tabular}{|c|c|c|c|c|c|c|c|}
\hline \#mach. & \#slices & \begin{tabular}{|l} 
gen. \& \\
comp. sim. \\
camp.
\end{tabular} & simulation & overall & speedup & efficiency & approach \\
\hline \multirow[t]{3}{*}{1} & \multirow[t]{3}{*}{1} & \multirow{3}{*}{$\begin{array}{c}0: 35: 55 \\
3: 4: 56\end{array}$} & \multirow{3}{*}{$\begin{array}{l}11242: 31: 28^{(*)} \\
13683: 20: 32^{(*)}\end{array}$} & $11243: 7: 23^{(*)}$ & $1.00 \times$ & $100.00 \%$ & dSLFV \\
\hline & & & & 13686:25:28 $8^{(*)}$ & $1.00 \times$ & $100.00 \%$ & rSLFV \\
\hline & & & & $+21.73 \%^{(*)}$ & $+0.00 \%$ & $+0.00 \%$ & overhead \\
\hline \multirow[t]{3}{*}{16} & \multirow[t]{3}{*}{128} & \multirow{3}{*}{$\begin{array}{c}0: 28: 56 \\
0: 30: 5\end{array}$} & \multirow{3}{*}{$\begin{array}{l}100: 17: 53 \\
348: 51: 47\end{array}$} & 100:46:49 & $111.56 \times$ & $87.16 \%$ & dSLFV \\
\hline & & & & $349: 21: 52$ & $39.18 \times$ & $30.61 \%$ & rSLFV \\
\hline & & & & $+246.66 \%$ & $+64.88 \%$ & $+\mathbf{5 6 . 5 5} \%$ & overhead \\
\hline \multirow[t]{3}{*}{32} & \multirow[t]{3}{*}{256} & \multirow{3}{*}{$\begin{array}{l}0: 28: 47 \\
0: 29: 18\end{array}$} & \multirow{3}{*}{$\begin{array}{c}57: 57: 27 \\
136: 18: 14\end{array}$} & $58: 26: 14$ & $192.40 \times$ & $75.16 \%$ & dSLFV \\
\hline & & & & $136: 47: 32$ & $100.05 \times$ & $39.08 \%$ & rSLFV \\
\hline & & & & $+134.08 \%$ & $+48.00 \%$ & $+36.08 \%$ & overhead \\
\hline \multirow[t]{3}{*}{64} & \multirow[t]{3}{*}{512} & \multirow{3}{*}{$\begin{array}{l}0: 28: 43 \\
0: 28: 50\end{array}$} & \multirow{3}{*}{$\begin{array}{c}26: 49: 4 \\
29: 23: 33\end{array}$} & $27: 17: 47$ & $411.89 \times$ & $80.45 \%$ & dSLFV \\
\hline & & & & $29: 52: 23$ & $458.15 \times$ & $89.48 \%$ & rSLFV \\
\hline & & & & $+9.44 \%$ & $-11.23 \%$ & $-\mathbf{9 . 0 3} \%$ & overhead \\
\hline
\end{tabular}

(b) Fuel Control System (

Table 4: Overall performance overhead (including disturbance trace generation and splitting, trace randomisation, computation of simulation campaigns and Simulink simulations) of [rSLFV with respect to to dSLFV (time in h:m:s).

(*) Estimated value. 


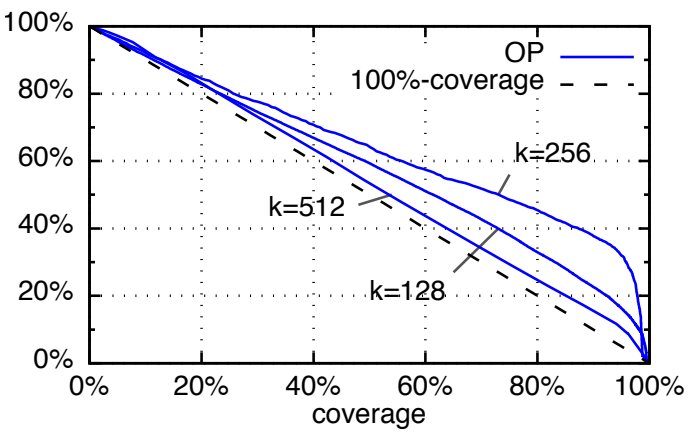

(a) $\mathrm{OP}$ against coverage

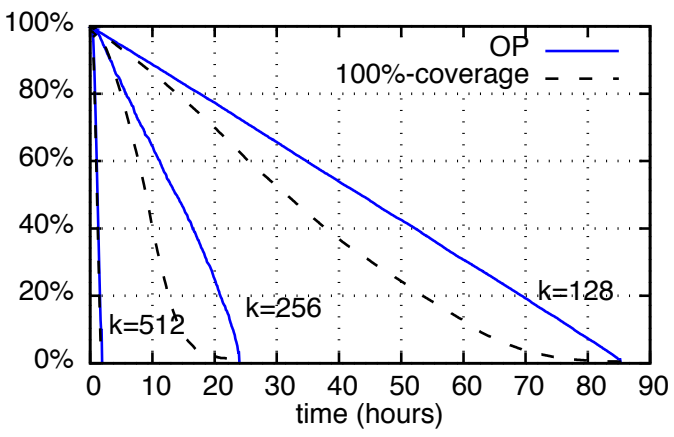

(b) $\mathrm{OP} \&$ coverage against time

Inverted Pendulum on a Cart (IPC), disturbance model $\mathcal{D}_{\text {IPC }}^{1}$

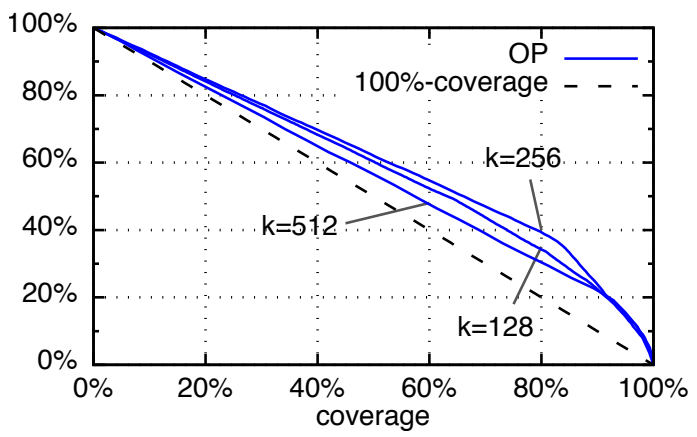

(c) $\mathrm{OP}$ against coverage

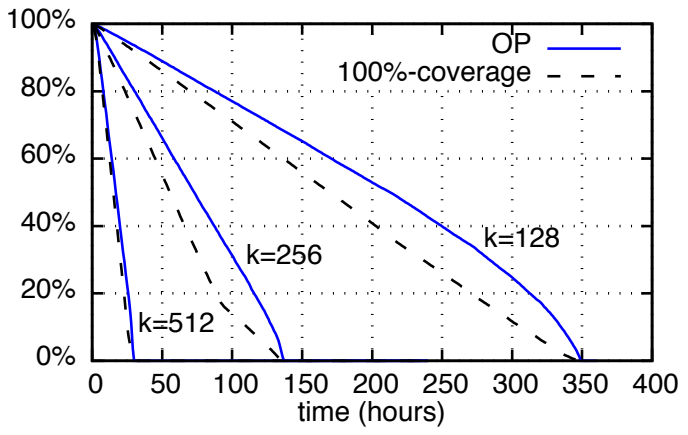

(d) $\mathrm{OP} \&$ coverage against time

Fuel Control System (

Figure 10: Omission Probability (OP) computation during the parallel execution of the simulation campaigns.

model checking. Since computation paths are not selected uniformly at random, 9] does not provide any information about the OP

Random model checking is a formal verification approach closely related to our setting. A random model checker provides, at any time during the verification process, an upper bound to the OP. Upon detection of an error, a random model checker stops returning a counterexample. Random model checking algorithms have been investigated, e.g., in [10, 22, 23. The main differences with respect to our approach are the following. (i) All random model checkers generate simulation scenarios using a sort of Monte-Carlo based random walk. As a result, unlike our algorithm, none of them is exhaustive (within a finite time horizon). (ii) Random model checkers (e.g., see [10]) assume availability of a lower bound to the probability of selecting (with a random-walk) an error trace. Of course, being exhaustive, we do not have any such assumption.

The coverage yielded by random sampling a set of test cases has been studied by 


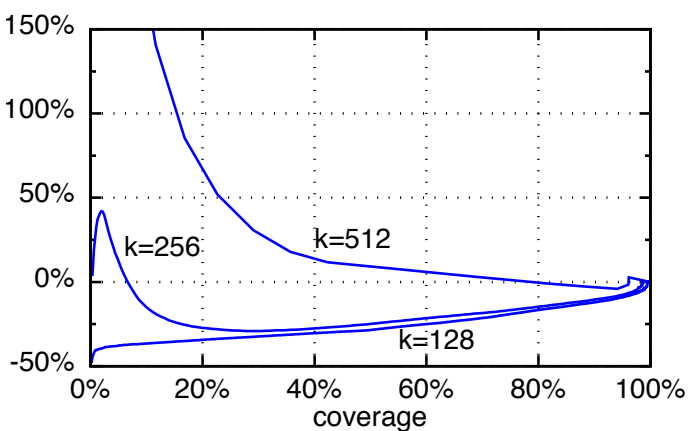

(a) Inverted Pendulum on a Cart (IPC), disturbance model $\mathcal{D}_{\text {IPC }}^{1}$

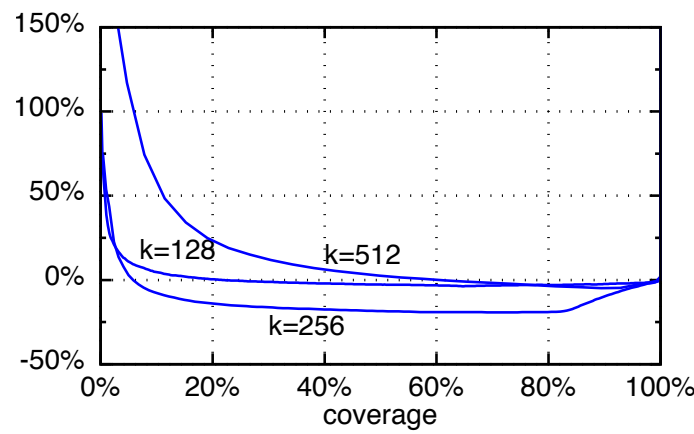

(b) Fuel Control System (FCS), disturbance model $\mathcal{D}_{\mathrm{FCS}}^{1}$

Figure 11: Completion time estimation error against coverage.

mapping it to the Coupon Collector's Problem (CCP) (see, e.g., 24]). In CCP elements are randomly extracted (uniformly and with replacement) from a finite set of $n$ test cases (disturbance traces in out context). Known results (see, e.g., 25]) tell us that the probability distribution of the number of test cases to be extracted in order to collect all $n$ elements has expected value $\Theta(n \log n)$, and a small variance with known bounds. This allows us to bound the $\mathrm{OP}$ during the verification. Differently from such $\mathrm{CCP}$. based approaches, here we not only bound the $\mathrm{OP}$ but also grant the completion of our verification task within just $n$ trials. This is made possible by the fact that we first generate all disturbance traces.

Monte-Carlo based robustness analysis of Cyber-Physical Systems (CPS;) has been investigated in 26. We note that, within a finite time bound, we are exhaustive whereas the approach in [26] is not. On the other hand, unlike our approach, [26] also evaluates how robustly the given property holds.

Probabilistic (e.g., see [27, 28) and, more specifically, simulation-based statistical model checking approaches (e.g., see [29, 30, 31, 32, 33, 16, 15, 34]) are closely related to our work. In particular, [16] addresses statistical model checking of Simulink models and presents experimental results on the very same Simulink case study we use here. The main differences between such approaches and ours are the following. (i) Probabilistic model checking is a white-box approach (a model is available), whereas we are in a black-box setting (only a simulator is available). Thus, only simulation-based statistical model checking approaches can be used in our context. (ii) Statistical model checking is not exhaustive (within a finite time horizon), whereas we are. (iii) Both probabilistic and statistical model checking use a stochastic model for the System Under Verification (SUV), whereas in our setting the SUV is deterministic and disturbances are nondeterministic. The probability measure in our context, as in random model checking, stems from the randomisation of the verification process itself. (iv) None of the available simulation-based statistical model checking approaches addresses the problem of the optimisation of the simulation campaign, which is an essential step to make our parallel random exhaustive Hardware In the Loop Simulation (HILS) based model checking 
viable.

Formal verification of Simulink models has been widely investigated, examples are in 35, 36, 37. Such methods however focus on discrete time models (e.g., Stateflow or Simulink restricted to discrete time operators) with small domain variables. Therefore they are well suited to analyse critical subsystems, but cannot handle complex system level verification tasks (e.g., our case studies). This is indeed the motivation for the development of statistical model checking methods as those in [15, 16, for the exhaustive HILS based approach in [8, and for our present parallel random exhaustive HILS based approach.

\section{Conclusions}

We presented a parallel random exhaustive Hardware In the Loop Simulation (HILS) based model checker for hybrid systems that, while being exhaustive with respect to the disturbance model given as input, provides at any time during the verification process an upper bound to the probability that the System Under Verification (SUV) exhibits an error in a yet-to-be-simulated scenario (Omission Probability, (OP).

Our experimental results on real world case studies from the Simulink distribution (namely: Inverted Pendulum on a Cart and Fuel Control System) show that, by exploiting parallelism, our approach to the computation of optimised simulation campaigns is feasible even for disturbance models entailing tens of millions of operational scenarios.

Also, simulation results show that, by exploiting parallelism, our simulation campaign optimiser effectively counteracts the simulation time overhead stemming from randomisation.

Finally, we have shown that our bound to the $\mathrm{OP}$ decreases about linearly with the coverage, which is as good as it can be even in the worst case scenario (just one error trace). Furthermore, resting on randomisation, we can use the coverage as a reliable estimator for the time needed to complete the verification process.

\section{Acknowledgments}

The research leading to these results has received funding from the European Union's 7th Framework Programme (FP7 2007-2013) under grant agreements n. 317761 and 600773. The authors would like to thank the anonymous reviewers, whose comments helped in improving this paper.

\section{References}

[1] T. Mancini, F. Mari, A. Massini, I. Melatti, E. Tronci, Anytime system level verification via random exhaustive hardware in the loop simulation, in: Proc. DSD 2014, IEEE, 2014.

[2] C. Baier, J. Katoen, Principles of Model Checking, MIT Press, 2008.

[3] Z. Yang, K. Hu, D. Ma, J.-P. Bodeveix, L. Pi, J.-P. Talpin, From \{AADL $\}$ to timed abstract state machines: A verified model transformation, Journal of Systems and Software 93 (2014) $42-68$. doi:http://dx.doi.org/10.1016/j.jss.2014.02.058

URL http://www.sciencedirect.com/science/article/pii/S0164121214000727 
[4] H. Mkaouar, B. Zalila, J. Hugues, M. Jmaiel, From aadl model to lnt specification, in: J. A. de la Puente, T. Vardanega (Eds.), Reliable Software Technologies Ada-Europe 2015, Vol. 9111 of Lecture Notes in Computer Science, Springer International Publishing, 2015, pp. 146-161. doi: 10.1007/978-3-319-19584-1_10 URL http://dx.doi.org/10.1007/978-3-319-19584-1_10

[5] E. Clarke, T. Henzinger, H. Veith, Handbook of Model Checking Springer, 2016. URL https : //books . google.it/books?id=qxG8oAEACAAJ

[6] R. Alur, Formal verification of hybrid systems, in: Proc. EMSOFT 2011, ACM, 2011, pp. $273-278$.

[7] E. M. Clarke, Jr., O. Grumberg, D. A. Peled, Model Checking, MIT Press, Cambridge, MA, USA, 1999.

[8] T. Mancini, F. Mari, A. Massini, I. Melatti, F. Merli, E. Tronci, System level formal verification via model checking driven simulation, in: Proc. of CAV 2013, Vol. 8044 of Lecture Notes in Computer Science, Springer, 2013, pp. 296-312.

[9] F. A. Aloul, B. D. Sierawski, K. A. Sakallah, Satometer:: how much have we searched? in: Proceedings of the 39th annual Design Automation Conference, DAC '02, ACM, New York, NY, USA, 2002, pp. 737-742. doi:10.1145/513918.514103 URL http://doi .acm.org/10.1145/513918.514103

[10] R. Grosu, S. Smolka, Monte carlo model checking, in: N. Halbwachs, L. D. Zuck (Eds.), Proc. TACAS 2005, Vol. 3440 of LNCS, Springer, 2005, pp. 271-286.

[11] E. Sontag, Mathematical Control Theory: Deterministic Finite Dimensional Systems, Texts in Applied Mathematics, Springer, 1998. URL http://books .google.it/books?id=ZFIp53GKSVMC

[12] T. Mancini, F. Mari, A. Massini, I. Melatti, E. Tronci, System level formal verification via distributed multi-core hardware in the loop simulation, in: Proc. PDP 2014, IEEE, 2014, pp. 734-742.

[13] G. Della Penna, B. Intrigila, I. Melatti, E. Tronci, M. Venturini Zilli, Exploiting transition locality in automatic verification of finite state concurrent systems, STTT 6 (4) (2004) 320-341. doi: 10.1007/s10009-004-0149-6.

[14] O. Maler, D. Nickovic, Monitoring temporal properties of continuous signals, in: Proc. FORMATS 2004 and FTRTFT 2004, Vol. 3253 of LNCS, 2004, pp. 152-166.

[15] E. M. Clarke, A. Donz, A. Legay, On simulation-based probabilistic model checking of mixed-analog circuits., Formal Methods in System Design 36 (2) (2010) 97-113. URL http://dblp.uni-trier.de/db/journals/fmsd/fmsd36.html\#ClarkeDL10

[16] P. Zuliani, A. Platzer, E. Clarke, Bayesian statistical model checking with application to simulink/stateflow verification, in: Proc. HSCC 2010, 2010, pp. 243-252.

[17] Y. J. Kim, M. Kim, Hybrid statistical model checking technique for reliable safety critical systems, in: Proc. ISSRE 2012, 2012, pp. 51-60.

[18] Y. J. Kim, O. Choi, M. Kim, J. Baik, T. Kim, Validating software reliability early through statistical model checking IEEE Software 30 (3) (2013) 35-41. doi:10.1109/MS.2013.24 URL http://doi.ieecomputersociety.org/10.1109/MS.2013.24

[19] P. Schrammel, D. Kroening, M. Brain, R. Martins, T. Teige, T. Bienmüller, Incremental bounded model checking for embedded software (extended version), CoRR abs/1409.5872. URL http://arxiv.org/abs/1409.5872

[20] MathWorks, Modeling a fault tolerant fuel control system, http://www.mathworks.com/help/ stateflow/examples/modeling-a-fault-tolerant-fuel-control-system.html

[21] T. Mancini, F. Mari, A. Massini, I. Melatti, E. Tronci, SyLVaaS: System Level Formal Verification as a Service, in: Proc. PDP 2015, IEEE, 2015.

[22] E. Tronci, G. Della Penna, B. Intrigila, M. Venturini Zilli, A probabilistic approach to automatic verification of concurrent systems, in: Proc. APSEC 2001, IEEE, 2001, pp. 317-324.

[23] H. Sivaraj, G. Gopalakrishnan, Random walk based heuristic algorithms for distributed memory model checking. Electr. Notes Theor. Comput. Sci. 89 (1) (2003) 51-67.

URL http://dblp.uni-trier.de/db/journals/entcs/entcs89.html\#SivarajG03

[24] A. Arcuri, M. Iqbal, L. Briand, Random testing: Theoretical results and practical implications, IEEE Transactions on Software Engineering 38 (2) (2012) 258-277. doi:10.1109/TSE.2011.121

[25] R. Motwani, P. Raghavan, Randomized Algorithms, Cambridge University Press, New York, NY, USA, 1995.

[26] H. Abbas, G. Fainekos, S. Sankaranarayanan, F. Ivančić, A. Gupta, Probabilistic temporal logic falsification of cyber-physical systems, ACM Transactions on Embedded Computing Systems 12 (2s) (2013) 95:1-95:30. doi : 10.1145/2465787.2465797 URL http://doi . acm .org/10.1145/2465787.2465797 
[27] G. D. Penna, B. Intrigila, I. Melatti, E. Tronci, M. V. Zilli, Finite horizon analysis of markov chains with the murphi verifier. STTT 8 (4-5) (2006) 397-409.

URL http://dblp.uni-trier.de/db/journals/sttt/sttt8.html\#PennaIMTZ06

[28] D. Jansen, J. Katoen, M. Oldenkamp, M. Stoelinga, I. Zapreev, How fast and fat is your probabilistic model checker? an experimental performance comparison, in: K. Yohav (Ed.), Hardware and Software: Verification and Testing, Proceedings of the Third International Haifa Verification Conference, HVC 2007, Vol. 4899 of Lecture Notes in Computer Science, Springer Verlag, London, 2008, pp. 69-85.

URL http://doc.utwente.nl/62646/

[29] H. L. S. Younes, R. G. Simmons, Probabilistic verification of discrete event systems using acceptance sampling, in: E. Brinksma, K. G. Larsen (Eds.), CAV, Vol. 2404 of Lecture Notes in Computer Science, Springer, 2002, pp. 223-235.

URL http://dblp.uni-trier.de/db/conf/cav/cav2002.html\#YounesS02

[30] H. L. S. Younes, Ymer: A statistical model checker in: K. Etessami, S. K. Rajamani (Eds.), CAV, Vol. 3576 of Lecture Notes in Computer Science, Springer, 2005, pp. 429-433.

URL http://dblp.uni-trier.de/db/conf/cav/cav2005.html\#Younes05a

[31] H. L. S. Younes, Probabilistic verification for "black-box" systems, in: K. Etessami, S. K. Rajamani (Eds.), CAV, Vol. 3576 of Lecture Notes in Computer Science, Springer, 2005, pp. 253-265. URL http://dblp.uni-trier.de/db/conf/cav/cav2005.html\#Younes05

[32] K. Sen, M. Viswanathan, G. Agha, On statistical model checking of stochastic systems, in: K. Etessami, S. K. Rajamani (Eds.), CAV, Vol. 3576 of Lecture Notes in Computer Science, Springer, 2005, pp. 266-280 URL http://dblp.uni-trier.de/db/conf/cav/cav2005.html\#SenVA05

[33] H. L. S. Younes, M. Z. Kwiatkowska, G. Norman, D. Parker, Numerical vs. statistical probabilistic model checking STTT 8 (3) (2006) 216-228.

URL http://dblp.uni-trier.de/db/journals/sttt/sttt8.html\#YounesKNP06

[34] A. David, K. G. Larsen, A. Legay, M. Mikučionis, Z. Wang, Time for statistical model checking of real-time systems, in: G. Gopalakrishnan, S. Qadeer (Eds.), Proceedings of the 23rd international conference on Computer Aided Verification (CAV), Vol. 6806 of LNCS, Springer-Verlag, Berlin, Heidelberg, 2011, pp. 349-355.

URL http://dl.acm.org/citation. cfm?id=2032305.2032332

[35] S. Tripakis, C. Sofronis, P. Caspi, A. Curic, Translating discrete-time simulink to lustre, ACM Trans. Emb. Comp. Syst. 4 (4) (2005) 779-818.

[36] B. Meenakshi, A. Bhatnagar, S. Roy, Tool for translating simulink models into input language of a model checker, in: Proc. ICFEM 2006, 2006, pp. 606-620.

[37] M. Whalen, D. Cofer, S. Miller, B. Krogh, W. Storm, Integration of formal analysis into a modelbased software development process, in: Proc. FMICS 2007, 2007, pp. 68-84. 


\section{A. List of Acronyms}

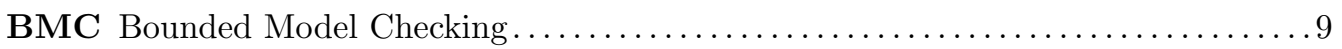

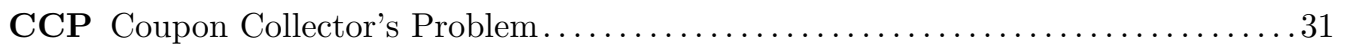

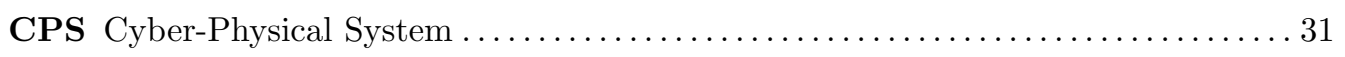

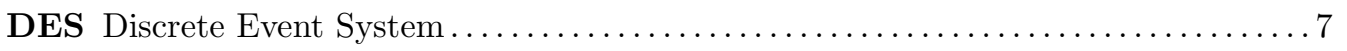

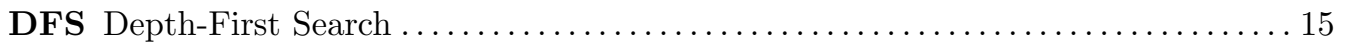

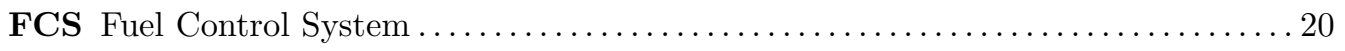

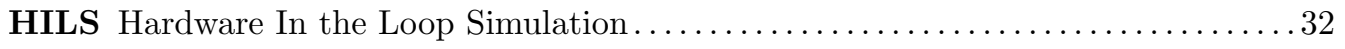

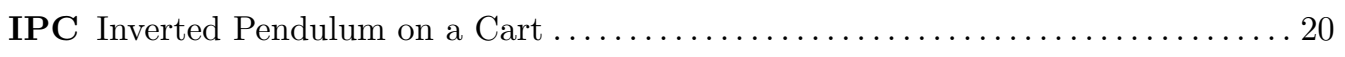

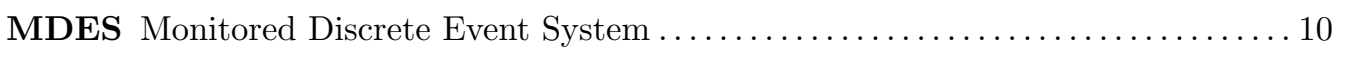

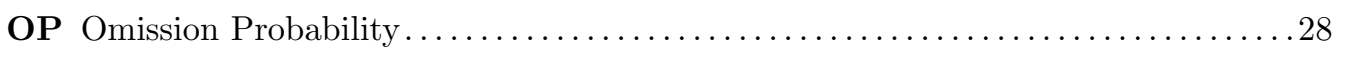

RSG Random Sequence Generator ..............................

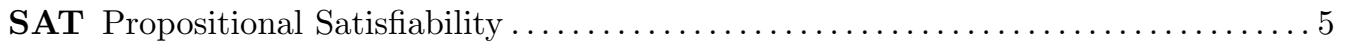

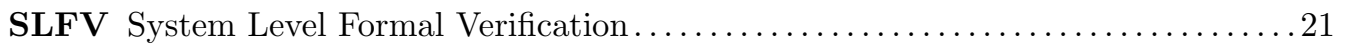

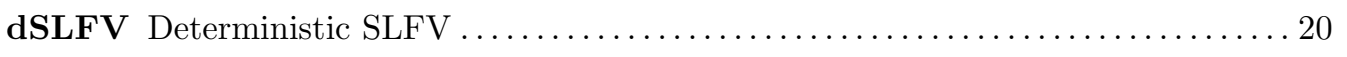

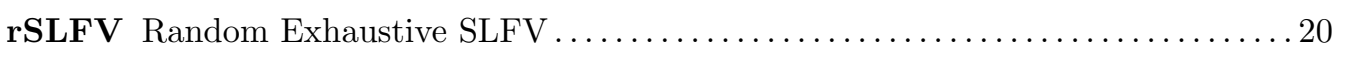

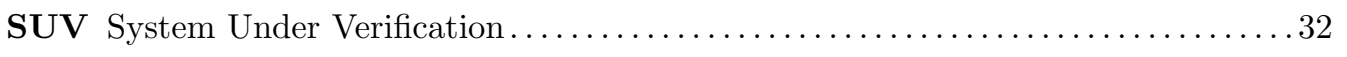

